\title{
600 eV Falcon-Linac Thomson X-ray Source
}

J. K. Crane, G. P. LeSage, T. Ditmire, R. Cross, K. Wharton, K. Moffitt, T. E. Cowan, G. Hays, V. Tsai, G. Anderson, R. Shuttlesworth, and $P$. Springer

\section{December 15, 2000}

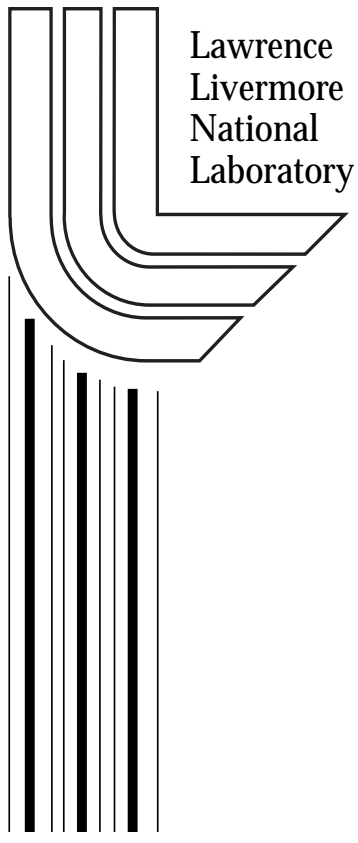


This document was prepared as an account of work sponsored by an agency of the United States Government. Neither the United States Government nor the University of California nor any of their employees, makes any warranty, express or implied, or assumes any legal liability or responsibility for the accuracy, completeness, or usefulness of any information, apparatus, product, or process disclosed, or represents that its use would not infringe privately owned rights. Reference herein to any specific commercial product, process, or service by trade name, trademark, manufacturer, or otherwise, does not necessarily constitute or imply its endorsement, recommendation, or favoring by the United States Government or the University of California. The views and opinions of authors expressed herein do not necessarily state or reflect those of the United States Government or the University of California, and shall not be used for advertising or product endorsement purposes.

This work was performed under the auspices of the U.S. Department of Energy by University of California, Lawrence Livermore National Laboratory under Contract W-7405-Eng-48. 


\section{INTRODUCTION}

The advent of $3^{\text {rd }}$ generation light sources such as the Advanced Light Source (ALS) at LBL, and the Advanced Photon Source at Argonne, have produced a revolution in x-ray probing of dense matter during the past decade. These machines use electronsynchrotrons in conjunction with undulator stages to produce $100 \mathrm{psec} x$-ray pulses with photon energies of several kiloelectronvolts $(\mathrm{keV})$. The applications for $\mathrm{x}$-ray probing of matter are numerous and diverse with experiments in medicine and biology, semiconductors and materials science, and plasma and solid state physics. In spite of the success of the $3^{\text {rd }}$ generation light sources there is strong motivation to push the capabilities of x-ray probing into new realms, requiring shorter pulses, higher brightness and harder x-rays. A $4^{\text {th }}$ generation light source, the Linac Coherent Light Source (LCLS), is being considered at the Stanford Linear Accelerator [1]. The LCLS will produce multi-kilovolt $\mathrm{x}$-rays of subpicosecond duration that are 10 orders of magnitude brighter than today's $3^{\text {rd }}$ generation light sources.[1] Although the LCLS will provide unprecedented capability for performing time-resolved $\mathrm{x}$-ray probing of ultrafast phenomena at solid densities, this machine will not be completed for many years. In the meantime there is a serious need for an ultrashort-pulse, high-brightness, hard x-ray source that is capable of probing deep into high-Z solid materials to measure dynamic effects that occur on picosecond time scales. Such an instrument would be ideal for probing the effects of shock propagation in solids using Bragg and Laue diffraction. These techniques can be used to look at phase transitions, melting and recrystallization, and the propagation of defects and dislocations well below the surface in solid materials. [2] These types of dynamic phenomena undermine the mechanical properties of metals and are of general interest in solid state physics, materials science, metallurgy, and have specific relevance to stockpile stewardship. Another x-ray diagnostic technique, extended x-ray absorption fine structure (EXAFS) spectroscopy, can be used to measure smallscale structural changes to understand the underlying atomic physics associated with the formation of defects. [2]

Through previous funding from LDRD and ER proposals we have undertaken the development of a Thomson x-ray source.[2] These Thomson x-rays will be produced by scattering a short-pulse, high-energy laser pulse off a $100 \mathrm{MeV}$ electron bunch produced by the LLNL linac in B194. To enable the production of a suitably short-pulse of x-rays, the linac must be seeded by a short-pulse, high-peak-current photoinjector that is synchronized to the laser system (the Falcon laser system in B194) that provides the femtosecond laser pulse. Figure 1 shows a conceptual picture of the Falcon-LinacThomson source. 


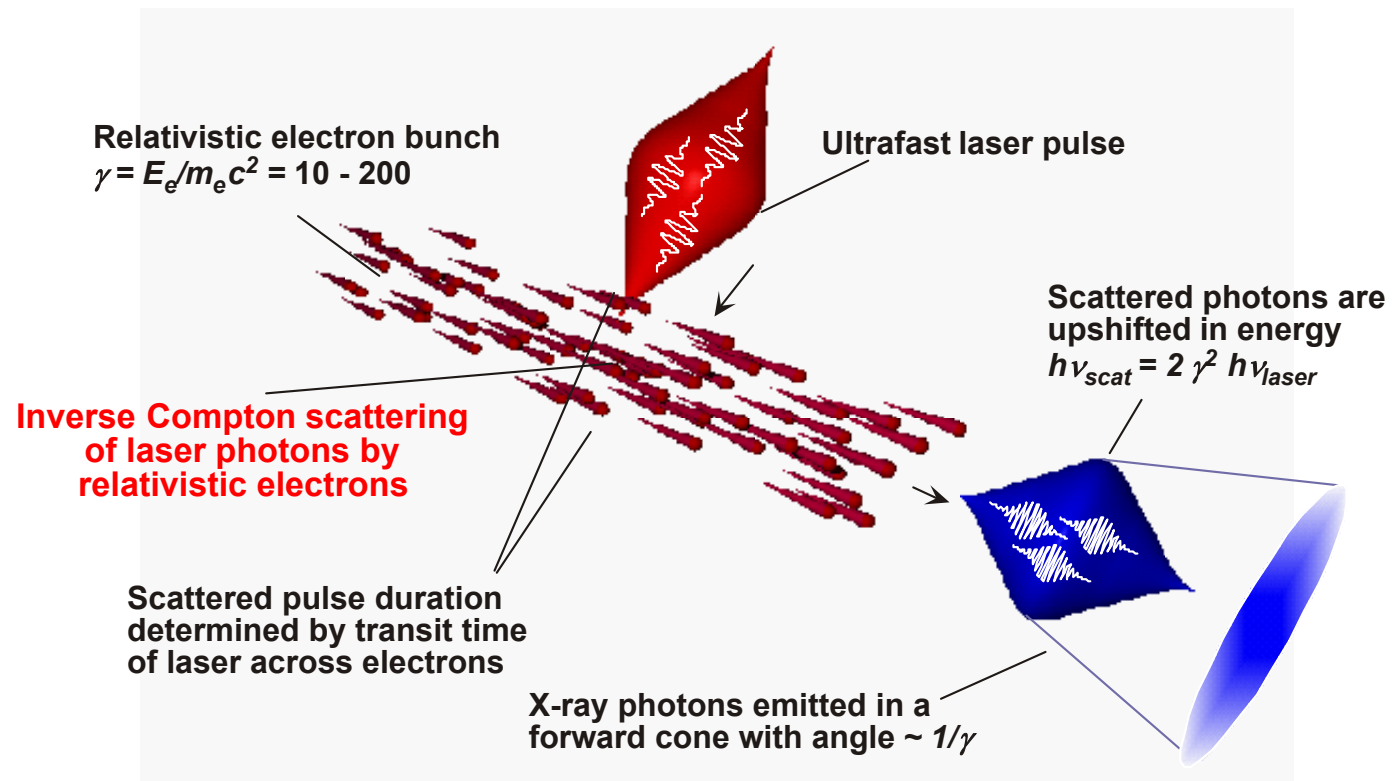

Figure 1. This figure shows the principle of short-pulse $\mathrm{x}$-ray production via Thomson scattering of visible photons from highly relativistic electrons.

In the past year we demonstrated the high-peak-current photoinjector, designed and built the photoinjector laser system, and synchronized the electron bunch with the Falcon laser system.[3] In the upcoming year we plan to upgrade the linac, adding computer controls, and replacing older rf drivers with newer sold-state devices; place the photoinjector on the linac beamline and demonstrate operation of the linac with the photoinjector as the electron seed; and complete the transportation of the full energy beam from the Falcon laser system to the Thomson interaction region. In this report we describe the photoinjector, the photoinjector laser system, and the results of an experiment to measure Thomson x-rays produced by scattering a portion of the photoinjector laser light from the $5 \mathrm{MeV}$ electrons produced by the photoinjector. This early demonstration of Thomson scattering serves several purposes: picosecond synchronization of the short-pulse laser to the photoelectrons, development of the x-ray detection and data acquisition capabilities, design and demonstration of a scaled, laserelectron interaction region, and integration of these tasks to produce and measure Thomson x-rays at $600 \mathrm{eV}$.

\section{PHOTOINJECTOR}

The LLNL Photoinjector uses the BNL/SLAC/UCLA 1.6 cell, standing wave accelerator geometry $[4,5]$ The photoinjector cavity and diamond-turned cathode were constructed using High Isostatic Pressure annealed OFHC Copper [6]. The photocathode surface has a measured flatness of $79 \mathrm{~nm}$ peak-to-valley over a 2 inch diameter $(\lambda / 8$ at $633 \mathrm{~nm})$. With the experience of the groups involved in the construction of previous photoinjectors, development of the LLNL photoinjector progressed quickly. First metal cutting for the photoinjector began in December of 1998 at the UCLA Physics department precision machine shop. High power RF was applied to the photoinjector in July of 1999. The first photoelectron beam was produced in January of 2000. 
In order to test the field profile of the photoinjector with the actual photocathode in place, a "bead-drop" was performed where a dielectric bead was hung from the end of a thin nylon line and lowered into the central axis of the photoinjector cavity. The usual technique requires a second test cathode plane to be made with a hole in the center for a string to pass through and suspend a bead in the horizontal direction: "bead-pull." The result of the bead-drop measurement is presented in Fig. 2, in comparison to the field profile generated by SUPERFISH[7], with a background image of the $\rho \mathrm{H}_{\phi}$ profile

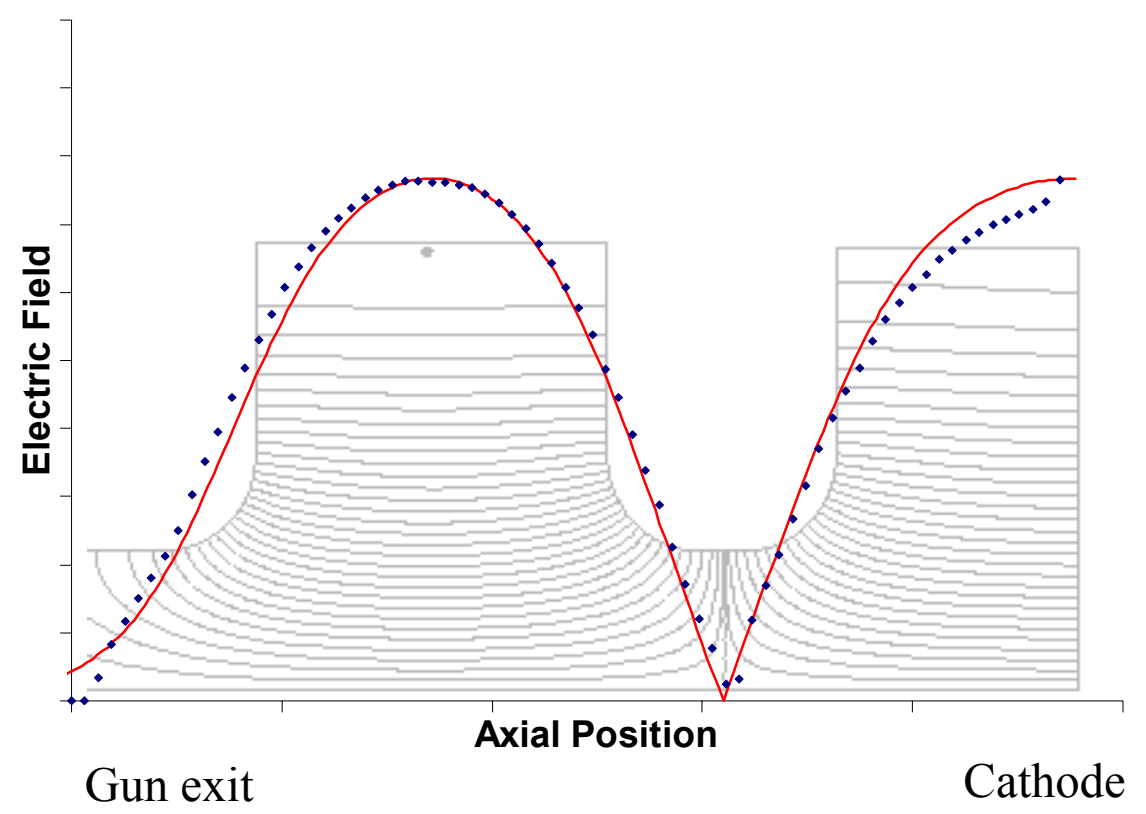

Figure 2. Bead-drop characterization of photoinjector field profile

The photoinjector is currently operated separately from the RF linac, and has been fully characterized using a diagnostic beamline. Characterization of the photoinjector has included measurement of cavity Q and filling time, dark current, charge and quantum efficiency, Schottky scan, energy, and emittance. Emittance has also been characterized as a function of charge and pulse length.

The energy range of field emitted electrons (dark current) in the cavity ranges broadly up to the peak beam energy. Characterization of the total field emission through current collection with a Faraday cup or integrating current transformer is subject to beam dynamics issues as the RF power and beam energy changes. An equivalent measurement of the Fowler-Nordheim macroscopic field enhancement factor $\beta$ can be made by measuring the radiation produced by the gun as a function of RF drive power [8]. A time-averaging radiation meter was placed at approximately half a meter from the photoinjector and measured radiation levels up to $500 \mathrm{mR} / \mathrm{h}$ with RF drive power up to 7 MW. An image of the dark current emission, focused by the photoinjector solenoids, and captured on a scintillator shows the emission from the cathode surface and the iris between the two gun cells in Fig. 3. 


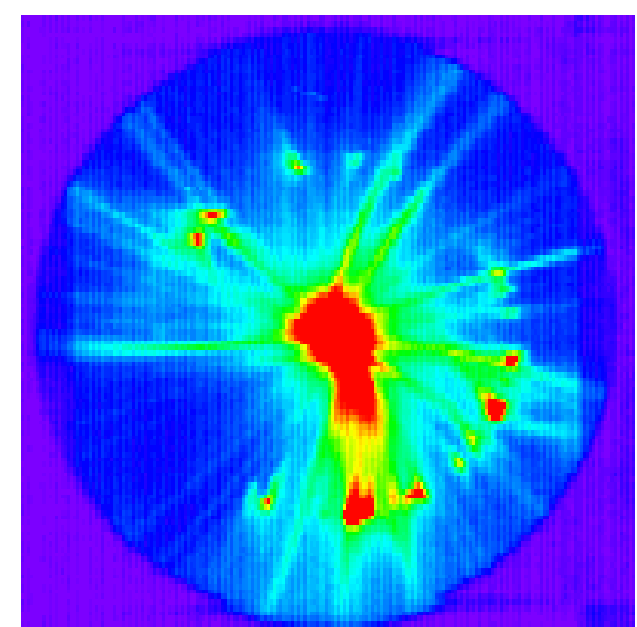

Figure 3. Dark current imaged with photoinjector solenoids

A plot of $1 / \mathrm{E}$ versus the logarithm of the dose rate divided by $\mathrm{E}^{4}$ is shown in Fig. 4, and provides the means of measuring the field enhancement factor $\beta$. The $\beta$ value measured for the LLNL HIP Copper photoinjector, taken from the slope of this plot was 62.

Fowler-Nordheim from Radiation

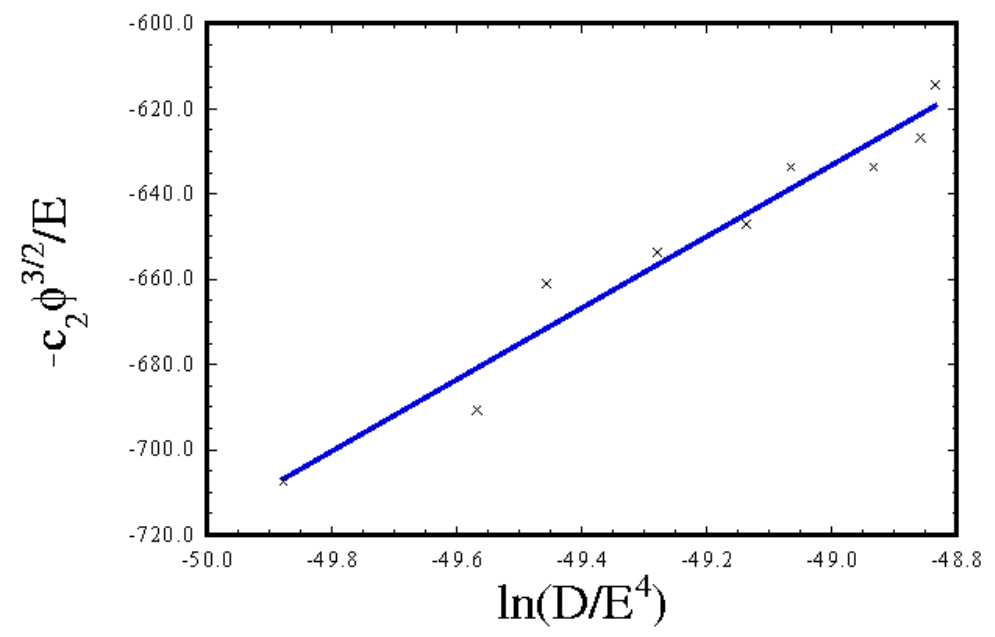

Figure 4. Fowler-Nordheim equivalent field emission plot.

The photoinjector cavity Ohmic Q was measured in cold test before and after brazing, and had a final cold-test value of 13,000. The value predicted by SUPERFISH, taking into account the loss of the copper surface including the cathode plane was 15,878 . The $\mathrm{Q}$ value of 13,000 was also verified by examining the filling time of the RF cavity with high power RF applied. The cavity fills with the characteristic $\mathrm{Q}_{0}$ and empties with $Q=\left[1 / Q_{0}+1 / Q_{e}\right]^{-1}=\frac{1}{2} Q_{0}$ as shown in Fig. 5. 


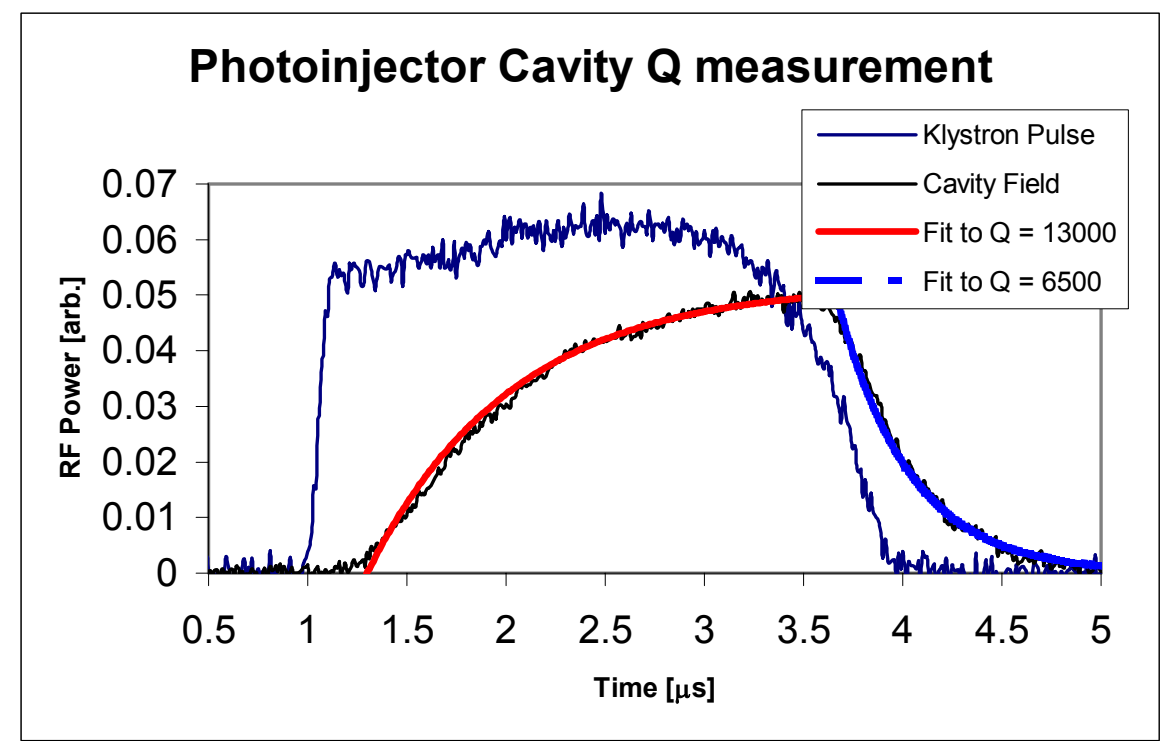

Figure 5. High-power measurement of photoinjector cavity $\mathrm{Q}_{0}$.

A summary of the LLNL photoinjector RF characteristics is presented in Table 1. The drive power and cathode peak field gradient correspond to production of a $5 \mathrm{MeV}$ photoelectron beam.

\begin{tabular}{ll}
\hline Beam parameter & Measured value \\
\hline Peak Cathode Gradient & $112 \mathrm{MV} / \mathrm{m}$ \\
Drive Power & $7.1 \mathrm{MW}$ \\
Ohmic Q & 13,000 \\
Cavity $\left|\mathrm{S}_{11}\right|$ & less than $-30 \mathrm{~dB}$ \\
Fowler-Nordheim, $\beta$ & 62 \\
\hline
\end{tabular}

Table 1. Photoinjector cavity parameters.

Photoelectron current was first measured on January 21, 2000. The program of beam measurements that followed this first beam production included Schottky scanning, in order to determine the injection phase for optimum emittance, and measurement of the total charge and quantum efficiency. An image of one of the first photoelectron beam profiles measured with the system is shown in Fig. 6. 


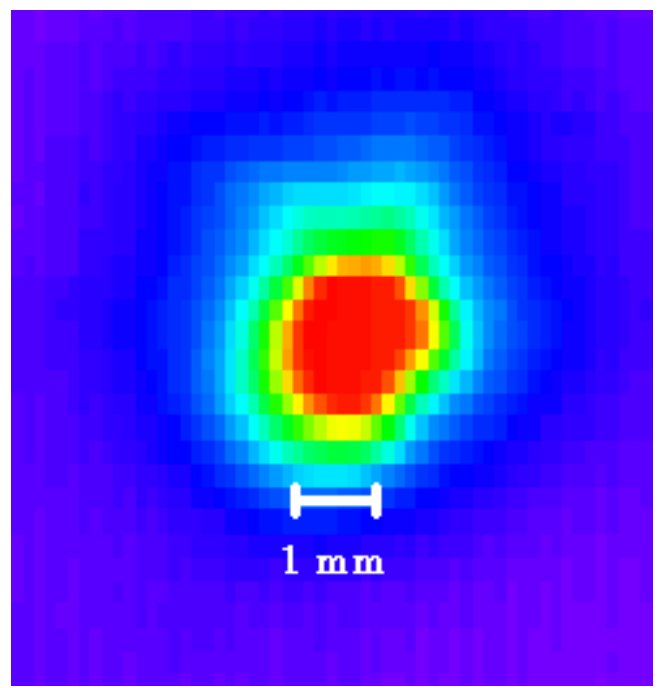

Figure 6. Photoelectron beam profile at $1 \mathrm{~m}$. from cathode.

The photoelectron bunch charge has been measured using both Faraday cups and a fast integrating current transformer. The peak charge per pulse that has been measured to date is $0.4 \mathrm{nC}$, and was purposely limited due to the small UV spot size on the photocathode. A full $\mathrm{nC}$ of charge extraction at high gradient with a small spot size would approach the region where cathode damage can result [9].

The quantum efficiency of the photoinjector operating at $23^{\circ}$ phase angle off of the RF crest was measured as $2.2 \times 10^{-5}$ using an integrating current transformer positioned directly at the photoinjector output, and a calibrated UV energy meter monitoring a split beam from the photocathode laser path. The required UV energy for $1 \mathrm{nC}$ of charge is thus $300 \mu \mathrm{J}$. The UV laser system has produced up to $400 \mu \mathrm{J}$ in its present configuration. The IR ( $805 \mathrm{~nm}$ wavelength) pulse length produced by the laser compressor is adjustable from 150 fs to more than $10 \mathrm{ps}$, giving a UV pulse length in the range from less than 100 fs to more than $9 \mathrm{ps}$. The electron pulse length has not been verified with a streak camera to date, and the electron pulse length is currently assumed to equal the UV pulse length.

The electron beam energy was measured using the steering magnets that are part of the diagnostic beamline. The steering magnet profile was integrated over a long longitudinal range extending into the fringe field region to give more accurate prediction of steering versus beam energy and magnet current. A linear fit of several magnet deflections, with variation of the RF drive power confirm a beam energy of $4.95 \mathrm{MeV}$ at the current RF drive power level of 7.1 MW.

The photoinjector electron beam emittance has been measured using a slit collimator [10]. This technique removes space charge from the beam measurement, and provides a single-shot means of measuring emittance through computer control and analysis. Launch phase and solenoid settings were optimized in real time using this diagnostic. The emittance as a function of beam launch parameters was characterized over a wide range of charge, pulse length, and beam radius on the photocathode. A schematic of the slit collimator arrangement is shown in Fig. 7. The collimator-slit widths are $50 \mu \mathrm{m}$, with collimator thickness is $5 \mathrm{~mm}$, and the drift length is $53 \mathrm{~cm}$. 


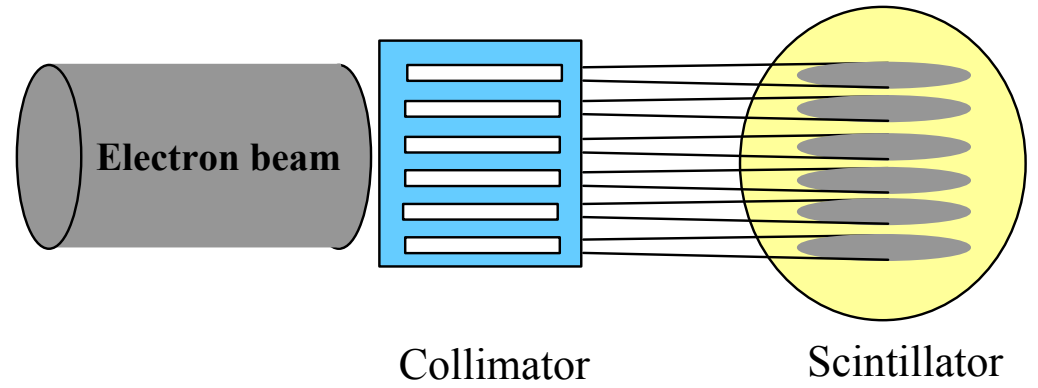

Figure 7. Collimator slit projection schematic.

An image of the photoelectron beam collected by the scintillator is shown in Fig. 8, and the virtual instrument panel for the emittance diagnostic is shown in Fig. 9.

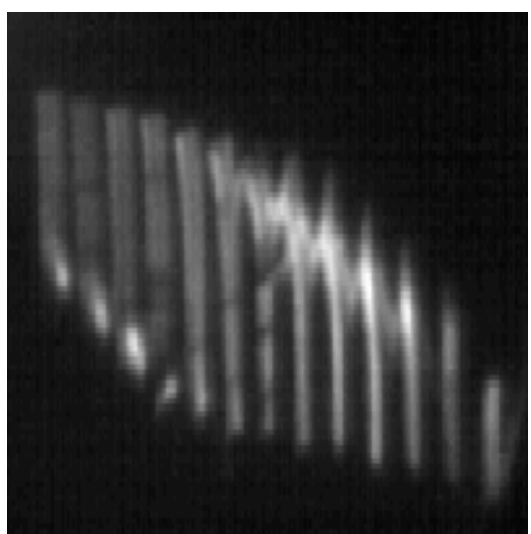

Figure 8. Collimator slit projection of photoinjector beam.

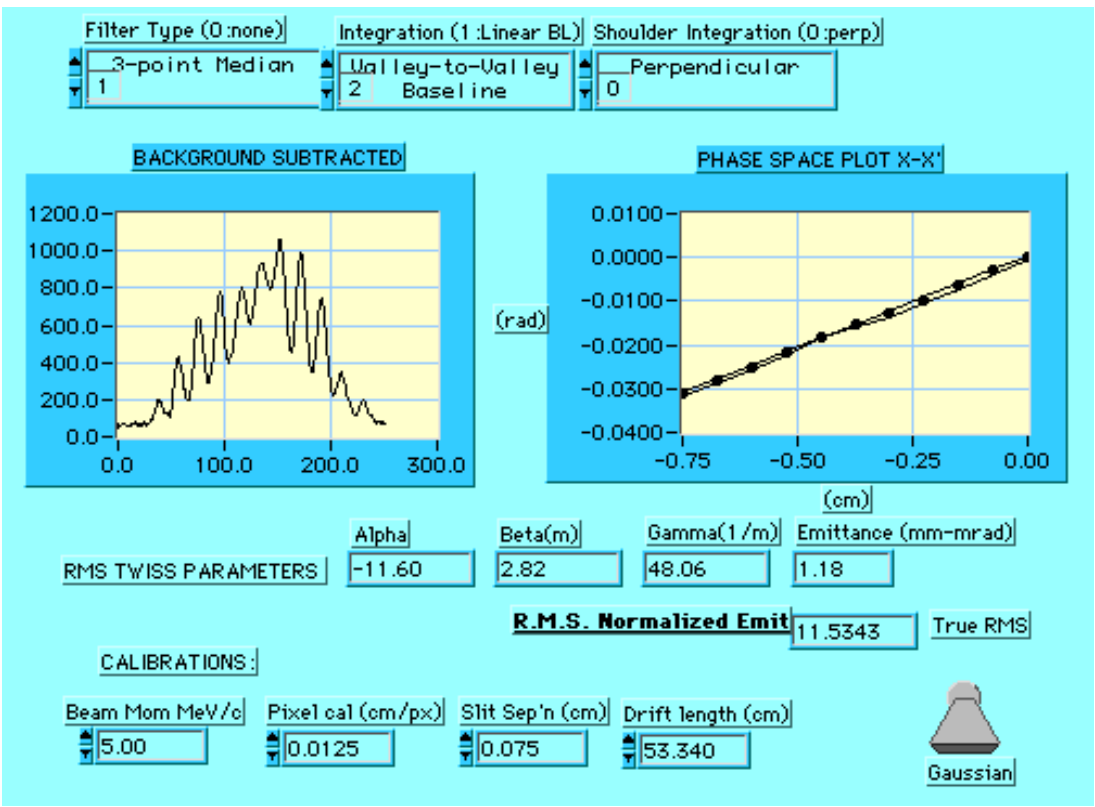

Figure 9. Slit emittance measurement virtual instrument panel.

The characteristics of the photoinjector for a specific pulse length are listed in Table 2. The measured emittance should be sufficient for the planned Thomson scattering source 
at $100 \mathrm{MeV}$, provided that excessive emittance growth through the linac is not encountered. The design value for the normalized emittance is $10 \pi \mathrm{mm}$-mrad, which implies a physical emittance at $100 \mathrm{MeV}$ of $\sim 51 \mu \mathrm{m}-\mathrm{mrad}$. With a $20 \mathrm{~cm}$ focal length final electron optic, and a $1 \mathrm{~mm}$ entrance spot radius ( $5 \mathrm{mr}$ convergence) the emittance limited spot size is $\sim 10 \mu \mathrm{m}$. Since the focal spot size of the FALCON laser beam with an $\mathrm{F} / 10$ optic is estimated to be $20 \mu \mathrm{m}$, the electron focal spot should overlap well with the laser spot.

\begin{tabular}{ll}
\hline & \\
\hline Beam parameter & Measured value \\
\hline Beam energy & $5 \mathrm{MeV}$ \\
UV laser pulse length & $7.1 \mathrm{ps} \mathrm{FWHM}$ \\
Bunch charge & $200 \mathrm{pC}$ \\
Emittance $_{\mathrm{x}, \mathrm{rms}, \mathrm{n}}$ & $5.8 \pi \mathrm{mm}-\mathrm{mrad}$ \\
Quantum efficiency $^{-5}$ & $2.2 \times 10^{-5}$ at $23^{\circ}$ off RF crest \\
\hline
\end{tabular}

Table 2. Photoinjector beam parameters.

\section{PHOTOINJECTOR LASER SYSTEM}

The photoinjector laser system (PLS) is used to generate photoelectrons for the photoinjector. This laser system is seeded by stretched pulses from the Falcon laser system so that the Linac can be synchronized to the Falcon master oscillator. The seed light is introduced into the regenerative amplifier ( regen ) of the photoinjector system via single-mode, polarization-preserving fiber that runs from the Falcon stretcher output to the B194 basement laboratory containing the PLS. Figure 10 shows a block diagram of the PLS integrated with the Falcon and Linac system in B194. Timing for the Falcon, Linac, and PLS derive from the Falcon master oscillator. In addition to the light pulse, timing signals from the Falcon timing rack are sent to the basement to trigger the YAG pump laser and regenerative amplifier Q-switch.

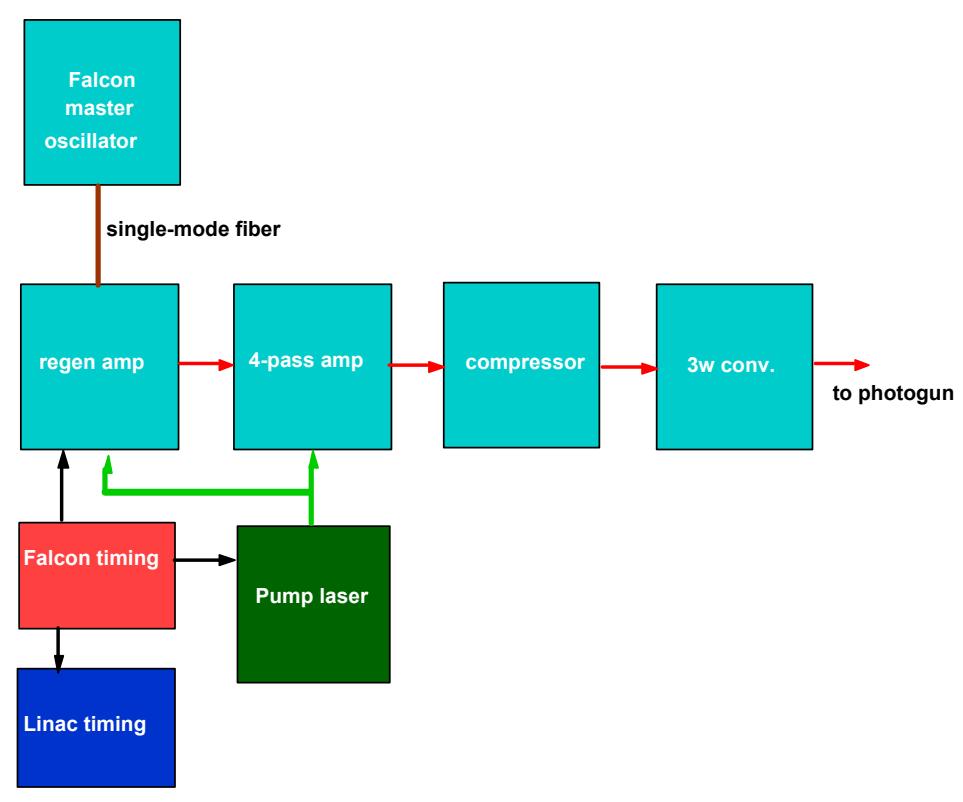


Figure 10. Block diagram of the Photoinjector Laser System (PLS) shows integration with Falcon and the Linac in B194.

The requirements for the PLS can be determined from the goal of obtaining $1 \mathrm{nC}$ from the photogun. If the photoelectron efficiency is $1 \mathrm{e}-5$ we need $6.25 \times 10^{14}$ photons at $266 \mathrm{~nm}$, or $0.47 \mathrm{~mJ}$ of $3 \mathrm{w}$ light at the photocathode. If the transmission from the tripling crystal to the copper cathode is $\sim 50 \%$ then we need $\sim 1 \mathrm{~mJ}$ of $3 \omega$ light at the frequency conversion crystal. If the output of the pulse compressor is $20 \mathrm{~mJ}$ then we need a conversion efficiency of $5 \%$. This value should be easily obtainable.

The PLS system consists of a fiber-seeded regenerative amplifier, a multipass power amplifier, a pulse compressor, and frequency conversion crystals for frequencytripling the $800 \mathrm{~nm}$ laser pulse. The two laser amplifiers, regen and multipass, are pumped by a single, frequency-doubled, Q-switched YAG laser that puts out $300 \mathrm{~mJ}$ of $532 \mathrm{~nm}$ light in a $8 \mathrm{~ns}$ pulse. Figure 11 shows a layout of the pump laser, regen, and multipass amplifiers. The output beam of the multipass amplifier is expanded and sent to the grating compressor

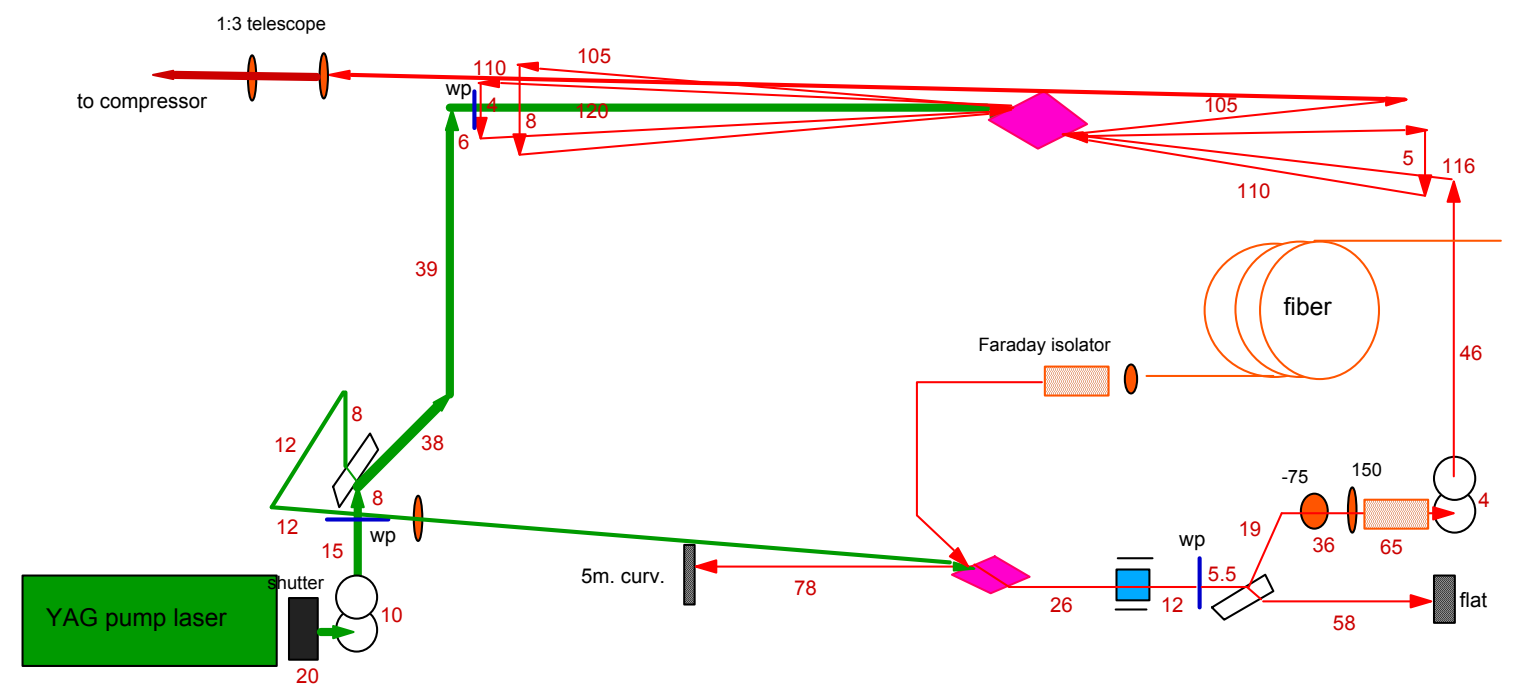

Figure 11. Layout shows YAG pump laser, seed, regenerative amplifier and 4-pass amplifier..

\section{A. Regenerative amplifier}

The regenerative amplifier ("regen") is a linear cavity that is Q-switched and cavitydumped using a single Pockels cell (PC) and PC driver in combination with an intracavity polarizer and waveplate. We use a Lasermetrics PC and quarter-wave driver. Initially, we seeded the regen by letting the seed pulse from the fiber reflect from the Ti-sapphire crystal face directly into the cavity, as is done on the Falcon regen. A major problem with seeding via reflection off the TiS crytal face is the $75 \%$ loss in seed energy due to the low reflectivity of the uncoated crystal. To inject more energy into the regen we added an optical directional coupler and inject the seed beam via a reflection from the cavity thinfilm polarizer as shown in Fig. 12. 


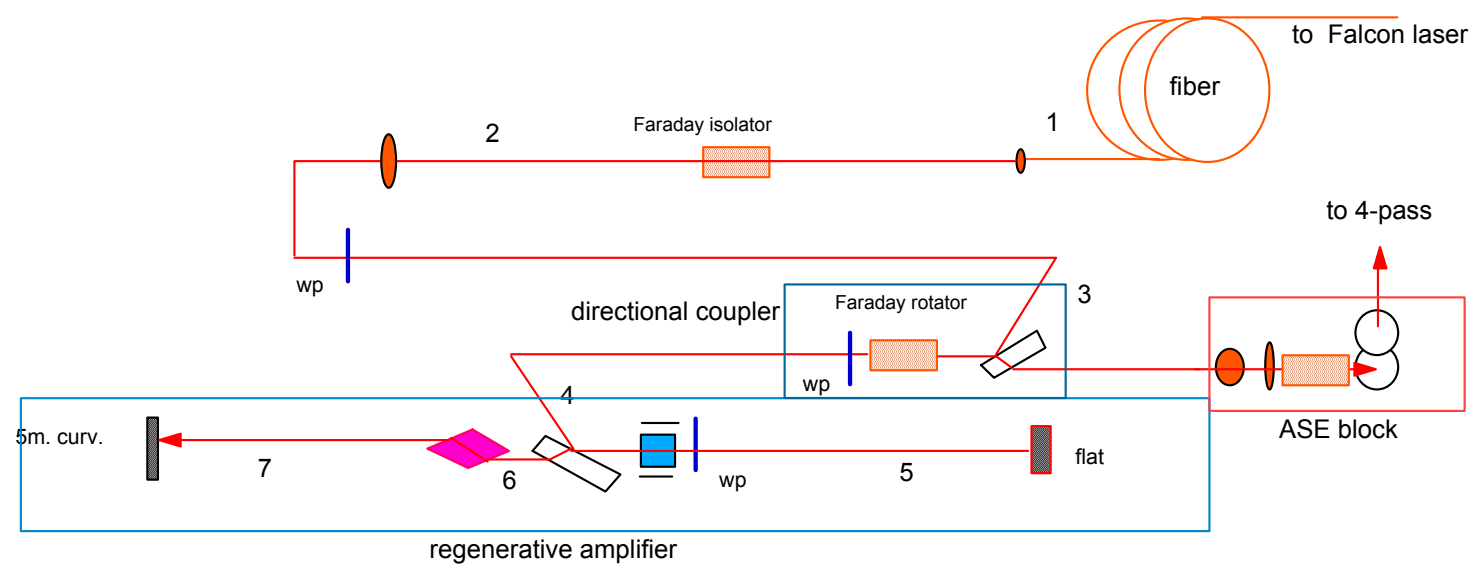

Figure 12. Layout shows regen, seed from fiber, directional coupler and ASE block.

We measured the propagation losses of the seed beam from the fiber output to the regen cavity. The transmission of the seed beam is summarized in the Table 3 :

$\begin{array}{ccc}\text { Location: } & \text { cw power }(\mathrm{mW}) & \text { Transmission } \\ 1 & 14 & 1.0 \\ 2 & 11 & 0.71 \\ 3 & 8.5 & 0.61 \\ 4 & 7.6 & 0.54 \\ 5 & 7.2 & 0.51 \\ 6 & 5.6 & 0.4 \\ 7 & 5.1 & 0.36\end{array}$

Table 3. Transmission loss between the seed fiber output and the regen cavity.

A cw seed power of $5.1 \mathrm{~mW}$ at $81 \mathrm{MHz}$ corresponds to a pulse energy of $63 \mathrm{pJ}$. In contrast we get less than a third of this energy if we introduce the seed as a reflection off the TiS crystal.

To trap the seed pulse in the cavity, the pulse double-passes a quarter-wave-plate, (QWP)), and unenergized PC, converting the s-polarized light to p-polarization. Immediately after passing the $\mathrm{PC}$ the driver is energized and subsequent passes see a full wave of retardation from the PC-QWP combination. The trapped pulse propagates in the cavity until the $\mathrm{PC}$ is switched off and the pulse exits the cavity via reflection from the thin-film-polarizer (TFP).

The regen cavity is a $1.8 \mathrm{~m}$ linear cavity with a $5 \mathrm{~m}$ curved end mirror and a flat mirror at the opposie end. We performed a paraxial analysis of the cavity to determine the stability, and the beam size and radius of curvature at the key locations in the cavity. The Paraxia model gives the beam size at the output TFP as $\mathrm{w}=0.88 \mathrm{~mm}\left(1 / \mathrm{e}^{2}\right.$ radius $)$ with a radius of curvature of $\mathrm{R}=5990 \mathrm{~mm}$. We use this information to model the propagation to the multipass amplifier and to determine the beam size on each pass. From the Paraxia model we get the following values for the $\mathrm{ABCD}$ matrix elements: $\mathrm{A}=-0.427, \mathrm{~B}=3.568 \mathrm{e}$, $\mathrm{C}=-4.0 \mathrm{e}-4, \mathrm{D}=1$. Using these values we calculate the product $\mathrm{g}_{1} \mathrm{~g}_{2}=(2+\mathrm{A}+\mathrm{D}) / 4=0.643$. This value of $g_{1} g_{2}$ satisfies the stability criterion: $0<g_{1} g_{2}<1$.[11] 
The seed mode from the fiber, $\mathrm{w}_{0}=4.0$ microns, is matched to the cavity eigenmode, $\mathrm{w}_{\mathrm{c}}=780$ microns using a simple two lens telescope. The advantage of using two lenses to mode-match is that the fiber position can be fixed and the waist size and location in the cavity can be adjusted by moving the two lenses. We use a $20 \mathrm{X}$ microscope objective in the fiber launch and a $300 \mathrm{~mm}$ f.l. lens placed about $600 \mathrm{~mm}$ from the objective. The microscope objective forms an image about $270 \mathrm{~mm}$ away for a magnification of $\sim 6.5 \mathrm{X}$. The second lens relays the first image to the flat cavity mirror for an additional magnification of $\sim 30$. To match the fiber mode to the cavity mode, we place two cameras from the Big Sky beam diagnostics system at either end of the cavity. We adjust the two lens positions plus point and center the seed into the cavity so that the seed beam and cavity mode overlap at both positions and are approximately the same size. Finally we checked the mode overlap at the output of the 4-pass amplifier, a distance $>6$ meters from the regen output.

Once the mode is spatially matched and overlapped we must set the switch in timing of the regen Pockels cell to cleanly trap a pulse in the cavity. The seed pulse at the input TFP is s-polarized. The pulse reflects from the TFP toward the flat cavity mirror. Initially the Pockels cell (PC) is turned off and the pulse is converted to p-polarization after double passing the quarter waveplate (QWP). The p-polarized pulse transmits through the TFP in the direction of the curved mirror. Once the pulse has cleanly passed, the $\mathrm{PC}$ is energized to produce a quarter wave of retardation. The pulse remains $\mathrm{p}$ polarized as long as the PC is "on", and is trapped in the cavity. When the pulse builds up to the desired energy level it is switched out of the cavity by turning off the PC.

After the turn-on time of the $\mathrm{PC}$ is set we can make a ringdown measurement to determine the actual loss in the cavity for the seed pulse. To measure the ringdown we measure the amplitude of the switched out pulse as a function of round trip at the regen output. Figure 13 shows these results. The points in the righthand plot are fitted to a power law relationship: $\mathrm{P}=\mathrm{T}^{\mathrm{x}}$ where " $\mathrm{T}$ " is the roundtrip transmission and " $\mathrm{x}$ " is the roundtrip index. The results show that the power initially falls off rapidly but slows to a higher transmission value of $\mathrm{T}=0.71$ after a couple of round trips. A more careful matching of the mode to the cavity might increase the transmission for the early round
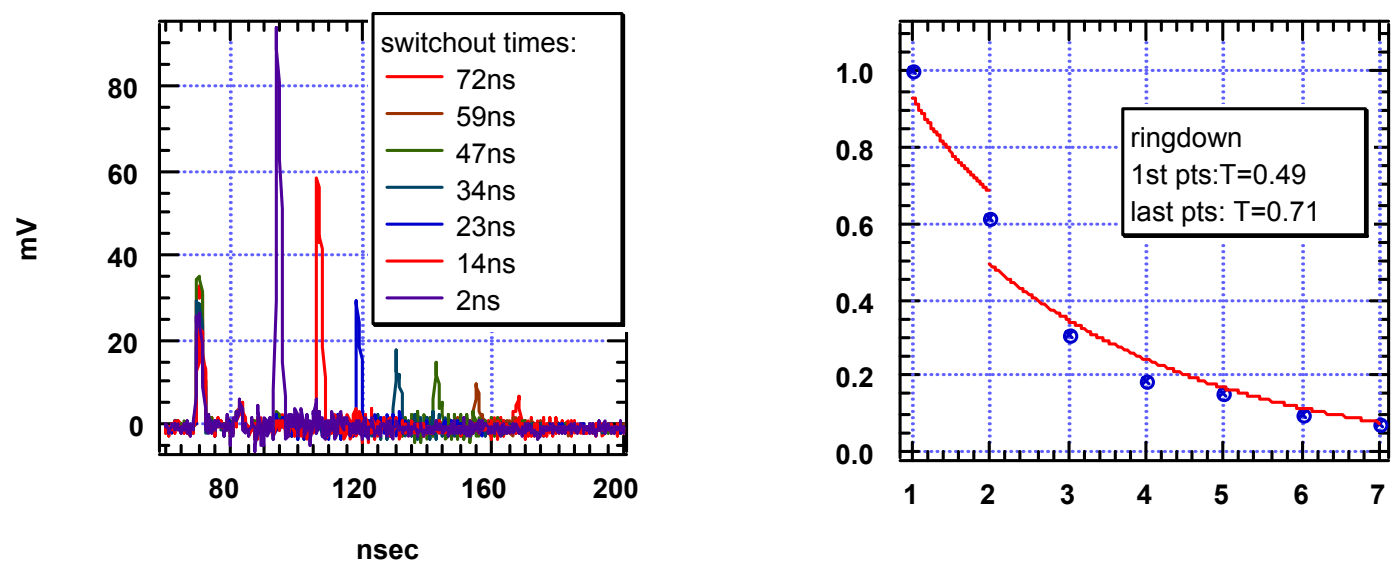

trips. 
Figure 13. Ringdown measurement in the regen cavity. Oscilloscope traces are shown in the lefthand plot; the pulse amplitude vs roundtrip is plotted on the right.

The regen showed a marked improvement in $\mathrm{S} / \mathrm{N}$ after proper seeding was completed. The improvement is noticeable in the cavity buildup and the output pulse as shown in Figure 14. The regen output pulse energy is 2-4 mJ for a pump energy of 52-60 $\mathrm{mJ}$.
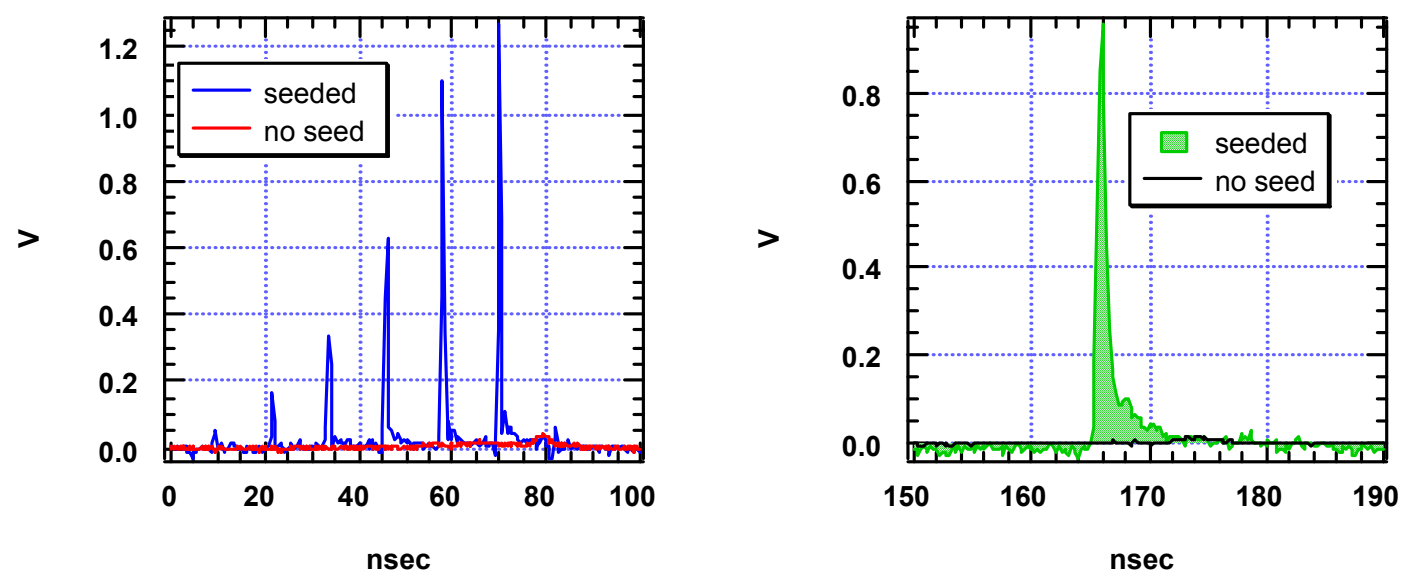

Figure 14. Left-hand plot shows the cavity buildup for the seeded and unseeded regen; righthand plot shows the output pulse for the seeded and unseeded regen.

\section{B. 4-pass amplifier}

A four-pass, bow-tie amplifier amplifies the $2 \mathrm{~mJ}$ regen output to $30-50 \mathrm{~mJ}$. The 4-pass is pumped with the 200-230 mJ of pump light from the same YAG pump laser that pumps the regen. A lens in the pump beam is used to adjust the pump beam size and produce an initial gain of $\sim 4-6$. The output of the regenerative amplifier is up-collimated using a 1:2 telescope to match the pumped cross-sectional area of the Ti-sapphire crystal in the 4-pass amplifier, $\mathrm{w}_{0} \sim 2 \mathrm{~mm}$. We placed a Faraday isolator at the output of the regen to prevent amplified spontaneous emission, ASE, from the 4-pass from propagating backward and being amplified by the regen ("ASE block" in Fig. 12). Figure 15 shows the layout of the 4-pass amplifier.

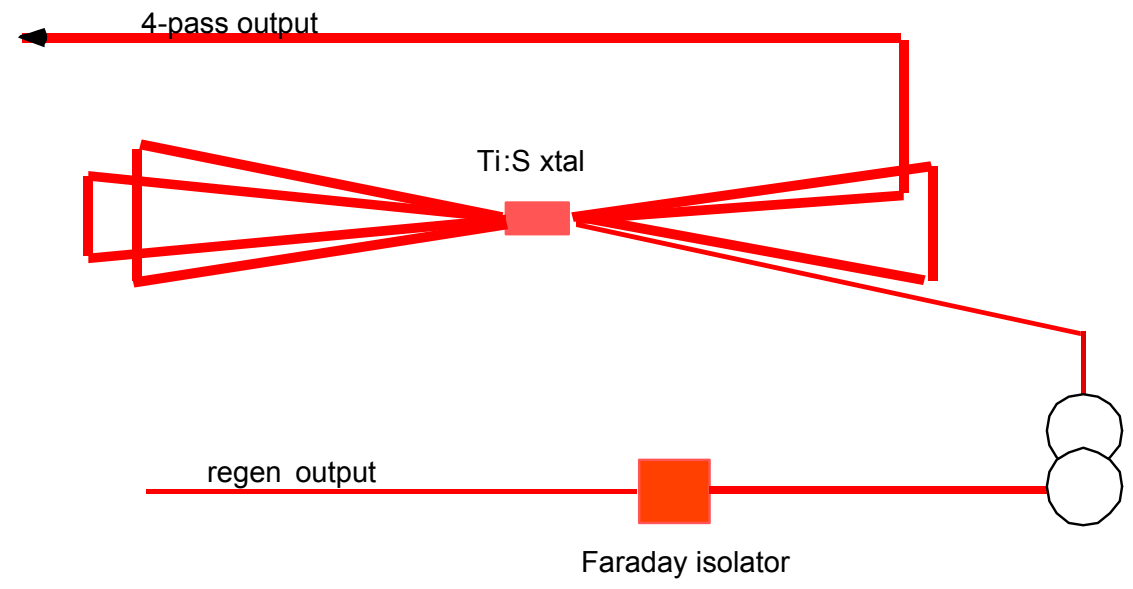


Figure 15. Four-pass layout with locations for transmission measurements

\section{$\underline{\text { C. Pulse compression and UV generation }}$}

The seeded output from the 4-pass is up-collimated by a 1:3 telescope to reduce the fluence below the damage threshold of the gold coatings on the compressor gratings. This beam is sent to a double-grating pulse compressor shown in the layout below. The gratings and four mirrors are gold coated and the compressor throughput was $\sim 40 \%$. The output of the compressor was sent either to an autocorrelator or down collimated by a 4:1 telescope as shown in Figure 16, and sent to the frequency conversion crystals.

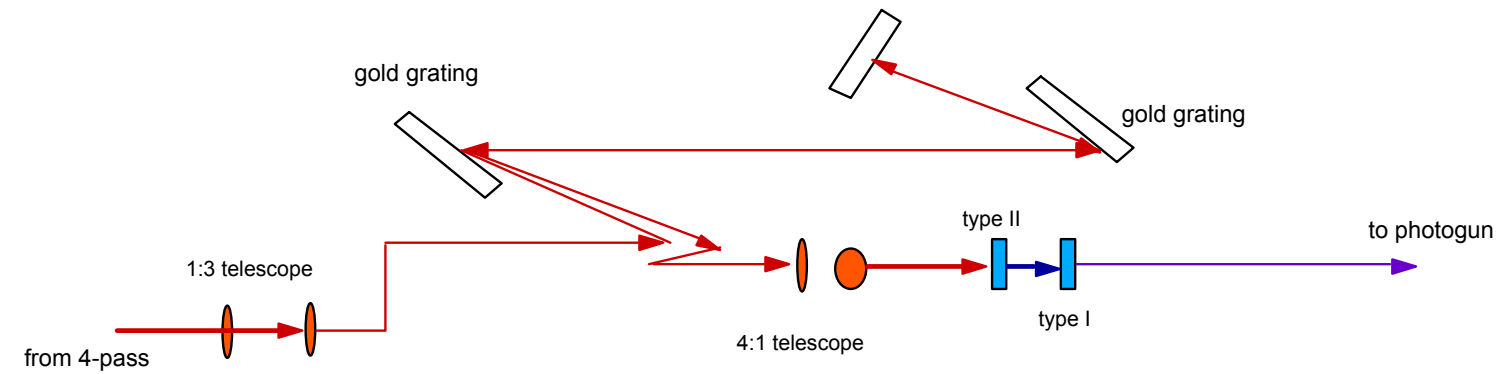

Figure 16. Layout of grating compressor and frequency tripling optics

The pulse out of the pulse compressor is down-collimated by a $4: 1$ telescope to increase the laser fluence for frequency conversion. The frequency conversion scheme is shown in the figure below. The fundamental at $800-820 \mathrm{~nm}$ is frequency doubled using a type II KDP crystal and the fundamental and second harmonic are mixed in a BBO crystal (type I) as shown in the figure.

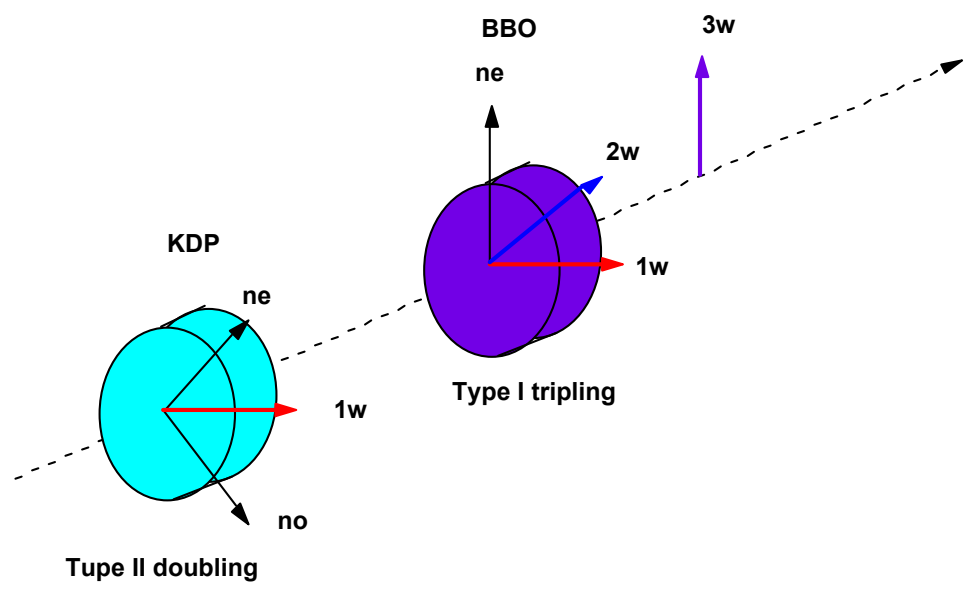

Figure 17 Tripling scheme for PLS laser system. 
The improved seeding of the regen increased the fraction of energy in the compressed pulse to $50 \%$. As a result we obtain $\sim 10 \mathrm{~mJ}$ of compressed light at the input to the doubling crystal. We measured $>2 \mathrm{~mJ}$ of frequency doubled light for $10 \mathrm{~mJ}$ input. We have measured $>0.5 \mathrm{~mJ}$ of $3 \mathrm{w}$ light at the photogun input. This gives a conversion efficiency $\mathrm{E}(3 \omega) / \mathrm{E}(1 \omega)=5 \%$.

Figure 18 shows the UV spot at a plane equivalent to the location of the cathode. For $300 \mu \mathrm{J}$ the maximum fluence in the spot is $\sim 50 \mathrm{~mJ} / \mathrm{cm} 2$. The beam shape is noticeably elongated as shown in the picture. We suspect that this elongated beam is caused by a combination of effects, including an oblong beam at the 4-pass output and nonuniform phase-matching. As we deliver more energy to the photocathode we may reshape the beam to make it rounder, larger, and more uniform. Reshaping the beam will lower the emittance of the photoelectron bunch and allow us to extract more photoelectrons from the gun.
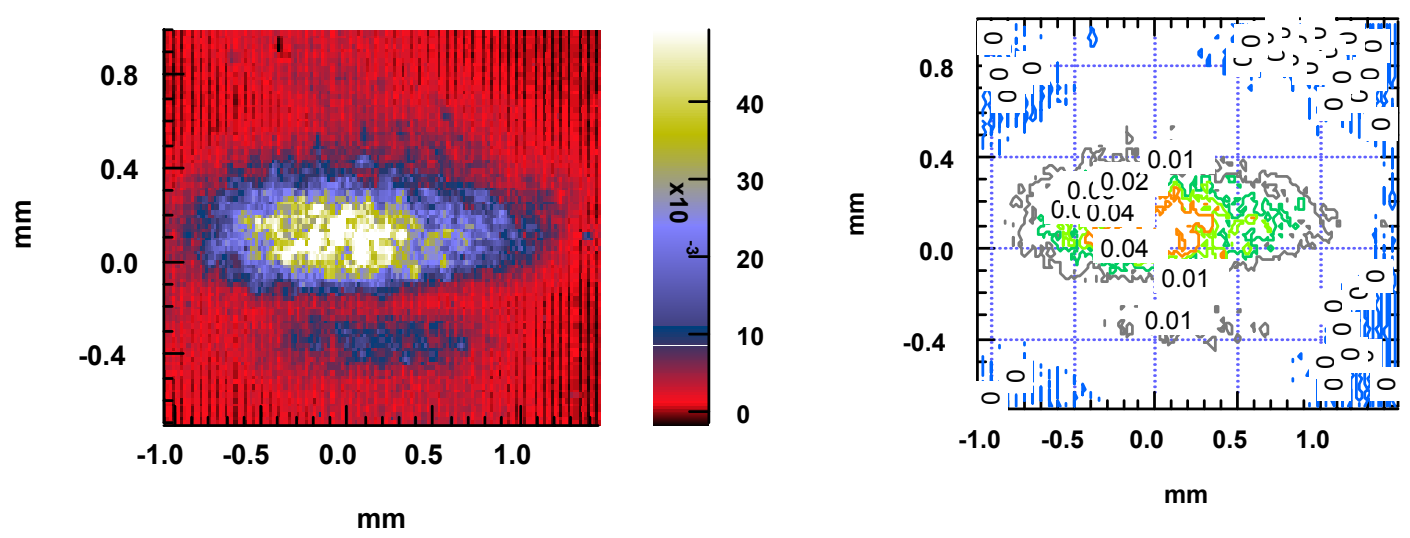

Figure 18. Images of the UV spot taken at the photocathode equivalent plane: figure on the right is an equal fluence contour map of the image on the left.

Table 4 lists a summary of the measured performance of the photoinjector laser system. The UV output energy at the photocathode, $\sim 0.5 \mathrm{~mJ}$ is barely adequate for producing $1 \mathrm{nC}$ of electronic charge in the beam. There are several improvements to the system that will increase the output energy and produce a larger electron bunch at the photocathode. These improvements include: add a slicer PC at the regen output to eliminate leakage ASE that is amplified in the 4-pass; replace three sets of uncoated telescope lenses with coated lenses to increase transmission; replace all gold mirrors in the compressor with dielectric mirrors; and relay image the pump beam from the YAG oscillator output coupler to the Ti:sapphire crystal. We plan to implement these improvements immediately. 


\begin{tabular}{ll}
\multicolumn{2}{c}{ Summary of results } \\
a. 4-pass output energy: & Eout $=30-57 \mathrm{~mJ}$ \\
b. 532 nm pump energy & Epump $=241 \mathrm{~mJ}$ \\
d. regen output energy & Eregen $=2.0-4.6 \mathrm{~mJ}$ \\
e. regen pump energy & Epump $=47-60 \mathrm{~mJ}$ \\
f. regen switchout time & Tsw $=240-270 \mathrm{~ns}$ (SRS setting) \\
g. transmission from regen to 4P input & $\mathrm{T}=52 \%$ \\
h. 4-pass transmission & $\mathrm{T}=23 \%$ \\
i. output energy from compressor & $\mathrm{E}=15-20 \mathrm{~mJ}$ \\
i. seeded energy @ 2w crystal & $\mathrm{E}=10 \mathrm{~mJ}$ \\
j. 2w energy @ 18 mJ input & $\mathrm{E}=2.2 \mathrm{~mJ}$ \\
k. delay between pump and regen output & $\mathrm{T}=442 \mathrm{~ns}$ \\
1. cw power at fiber output & $\mathrm{P}=10-14 \mathrm{~mW}$ \\
m. cw seed power in cavity & $\mathrm{P}=5.1 \mathrm{~mW}$ \\
n. energy in seed pulse in cavity & $\mathrm{E}=63 \mathrm{pJ}$ \\
n. 3w energy @ photogun window & $\mathrm{E}=0.5 \mathrm{~mJ}$ \\
o. compressed pulse length @ $820 \mathrm{~nm}$ & $150 \mathrm{fs}-\mathrm{several} \mathrm{ps}$ \\
p. $3 w$ center frequency & $269 \mathrm{~nm}$
\end{tabular}

Table 4 Current performance of the photoinjector laser system.

\section{PULSEWIDTH AND SPECTRAL MEASUREMENTS}

The characteristics of the electron beam produced by the photoinjector are critically dependent on the characteristics of the UV laser pulse that illuminates the $\mathrm{Cu}$ cathode of the gun. The energy of the UV photons from the photoinjector laser must surpass the work function of the $\mathrm{Cu}$ cathode. The rf field that pulls electrons from the surface must be accurately timed to the laser pulse arrival at the cathode. The laser beam energy determines the amount of charge emitted by the cathode. The laser pulse shape in area and temporal width determine the space charge and emittance of the electron pulse. In this section we summarize the results of our pulse width and related measurements for the photoinjector laser system. 


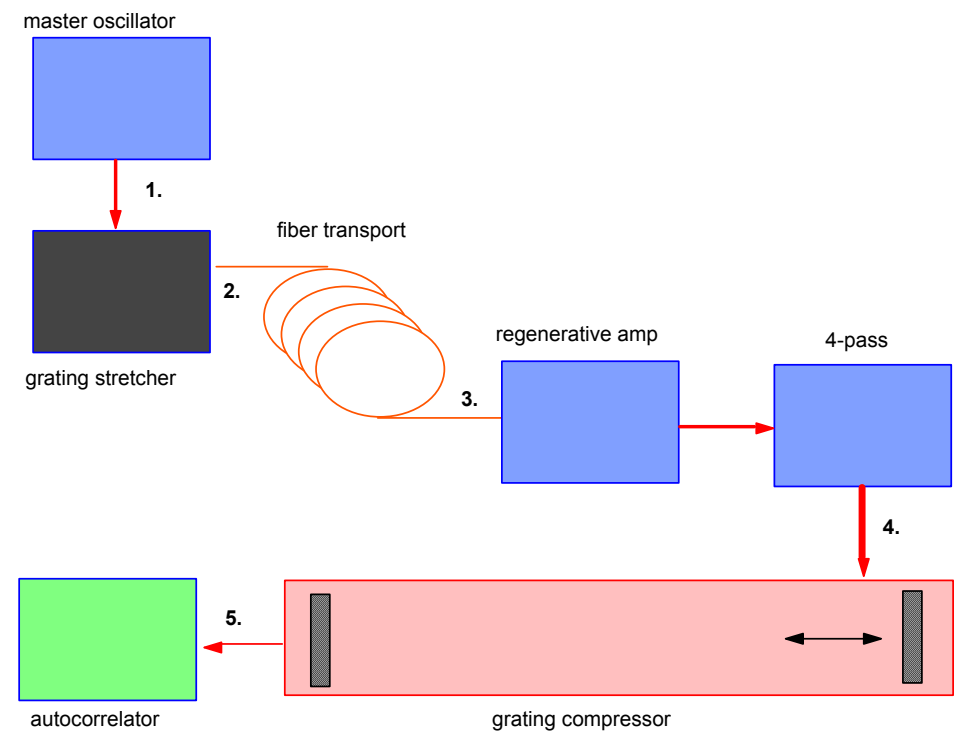

Figure 19. Block layout of the Falcon/Photoinjector laser showing various measurement points.

Figure 18 shows a block layout of the Falcon master oscillator and grating stretcher, the fiber link to the photoinjector laser, and the photoinjector layout. The numbers in the drawing indicate the locations where we made spectral and temporal measurements of the laser pulse. In this section we describe some of the details of our pulse shape and spectral measurements, plus a model that predicts pulse width versus compressor grating spacing.

\section{A. Spectral measurements}

We measured the spectrum of the laser pulse at three different locations: the Falcon master oscillator output, the fiber transport output in the basement, and the photoinjector 4-pass amplifier output, which is the input to the grating compressor. The measurements were made with a SPEX 1/4 meter spectrometer with a Cohu camera as the detector. The spectrometer was calibrated with a Kr lamp. Figure 20 shows lineouts from the camera images for the three different locations. 


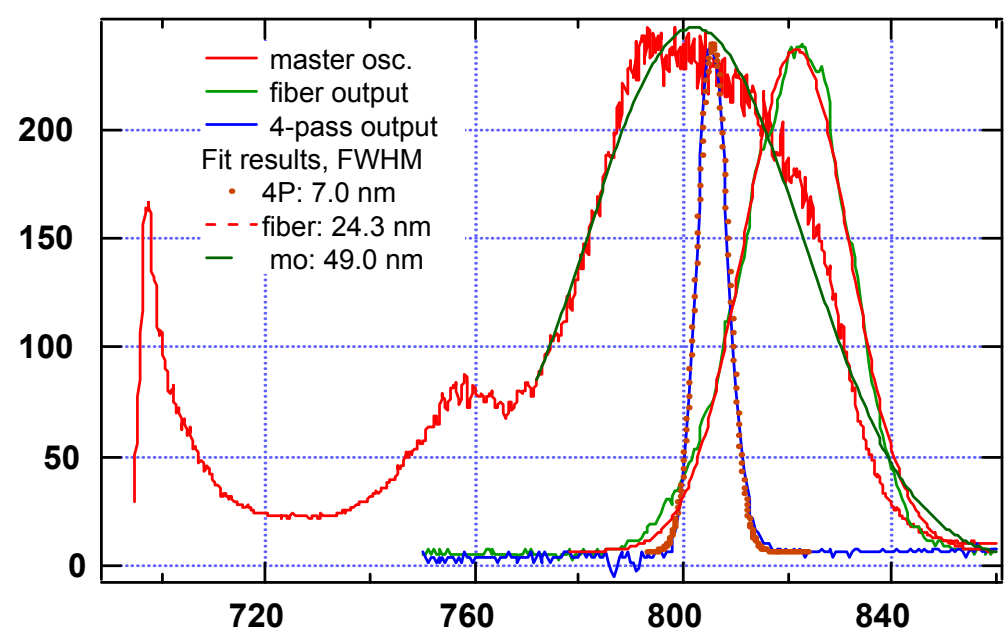

Figure 20. Spectra taken at the master oscillator, fiber output and 4-pass outputs.

We fit each spectrum to a Gaussian shape and determined the full-width-at halfmaximum, FWHM. The results along with conversion to frequency bandwidth, BW and time-bandwidth-limited pulse width are given in Table 5.

\begin{tabular}{lllll} 
Measurement location & $\lambda_{0}, \mathrm{~nm}$ & $\Delta \lambda, \mathrm{nm}$ & $\Delta v, \mathrm{THz}$ & $\mathrm{Tp}, \mathrm{fs}$ \\
\hline 1. $\quad$ master oscillator & 802 & 49 & 22.8 & 21.9 \\
3. fiber output & 822 & 24.3 & 10.8 & 46.3 \\
4. $\quad$ 4-pass output & 806 & 7.0 & 3.2 & 154
\end{tabular}

Table 5. Summary of spectral measurements for photoinjector laser.

The results for the spectral linewidth provide important input for modeling the temporal pulse width as a function of grating spacing, which ultimately provides values for the $3 \mathrm{w}$ laser pulse length illuminating the photocathode since we do not have a good direct measurement.

\section{B. Pulse width measurements}

We measured the pulse width of the Falcon/Photoinjector laser system at all 5 locations shown in Fig. 19. The techniques for these five pulsewidth measurements are summarized in Table 6.

Measurement location

1. master oscillator output

2. stretcher output

3. fiber transport output

4. 4-pass output

5. compressor output technique

cw autocorrelator

sampling scope

sampling scope

transient digitizer

single-shot autocorrelator measured pulsewidth

$24 \mathrm{fs}$

600 ps

843 ps

184 ps

$>150$ fs 
Table 6. Summary of results for pulsewidth measurements on Falcon/Photoinjector laser system.

C. Pulsewidth of master oscillator

We measured the pulsewidth of the master oscillator using the onboard autocorrelator diagnostic that is part of the Femtosource laser. Figure 21 shows the recorded oscilloscope trace for the autocorrelation. We measure a FWHM of 14 cycles, yielding a pulse width of

$$
\mathrm{T}_{\mathrm{p}}=14 *(2.67 \mathrm{fs} / \text { cycle }) / 1.55=24 \mathrm{fs}
$$

The factor, 1.55, accounts for the autocorrelation of a $\operatorname{sech}^{2}$ pulse. [12]

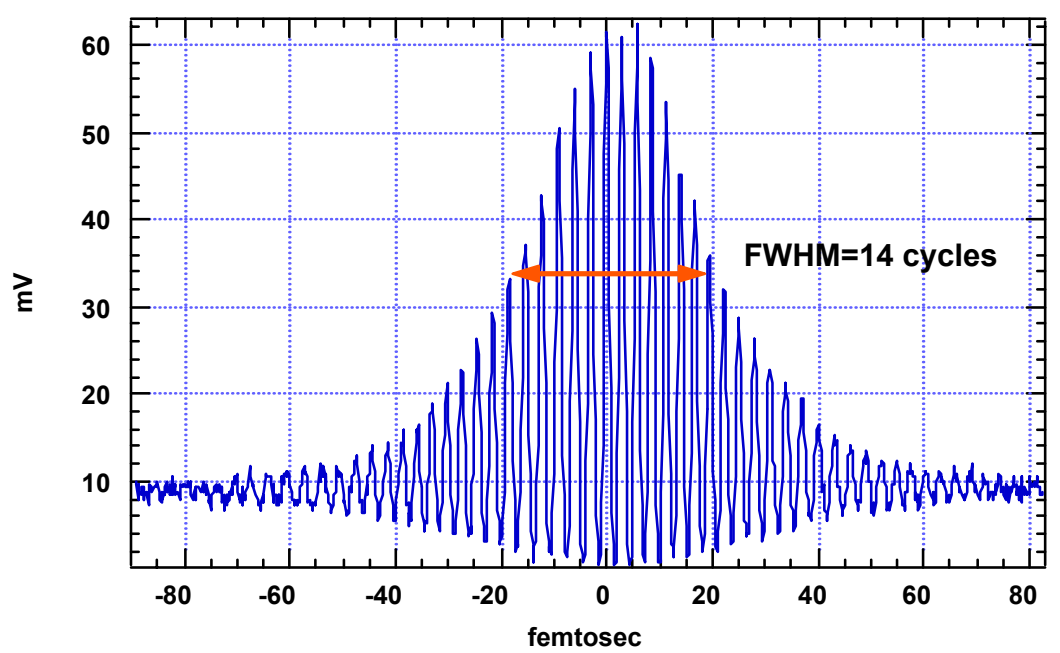

Figure 21. $\mathrm{Cw}$ autocorrelation of the master oscillator.

The pulse at the output of the grating stretcher in Falcon was measured at an earlier date with a sampling scope. The data for this measurement is not available, but the claim is that the measured pulsewidth at the output of the stretcher is $600 \mathrm{ps}$.

\section{Pulsewidth at the output of the fiber transport to photoinjector laser}

We used a Tek 11801A sampling scope and a NewFocus model 1431 InGaAs photodiode to measure the $81 \mathrm{MHz}$ pulse train at the output of the fiber that connects Falcon to the basement photoinjector laser. Figure 22 shows an average of 4 pulses, taken to accomodate the jitter in the pulse train due to cavity fluctuations in the master oscillator cavity. A Gaussian fit to the data yield a FWHM of 843 ps for the pulsewidth. The pulse is lengthened slightly as it travels through the fiber due to the fiber's dispersion. 


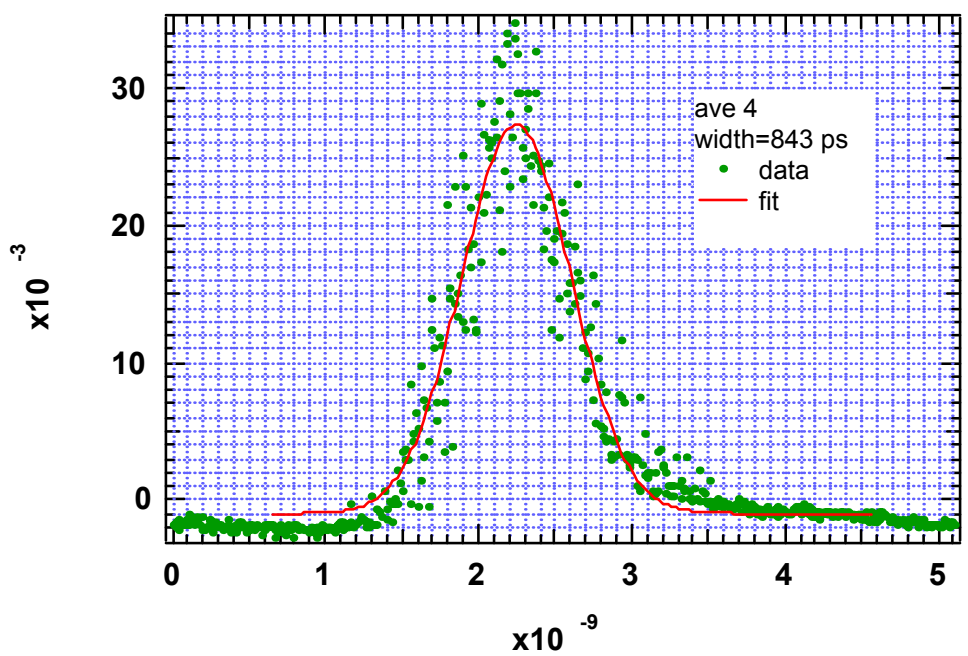

Figure 22. Measured pulse at the output of the fiber link between Falcon and photoinjector laser.

E. Pulsewidth at the output of the photoinjector laser 4-pass amplifier

The regenerative amplifier on the photoinjector laser was designed to pull the output toward shorter wavelength so that the $3 \mathrm{w}$ photons would be energetic enough to excite the $\mathrm{Cu}$ valence electrons. As a result the bandwidth of the regen is smaller than the other systems and the reduced bandwidth shortens the stretched pulse considerably. Since the regen operates at $10 \mathrm{~Hz}$ we used an SCD5000, $4.5 \mathrm{Ghz}$ transient digitizer and a Hamamatsu R1328U vacuum photodiode to measure the pulse shape. The photodiode has a 60 ps risetime and a 55 ps falltime. Figure 23 shows pulse measurements taken at the 4pass output and the compressor output.
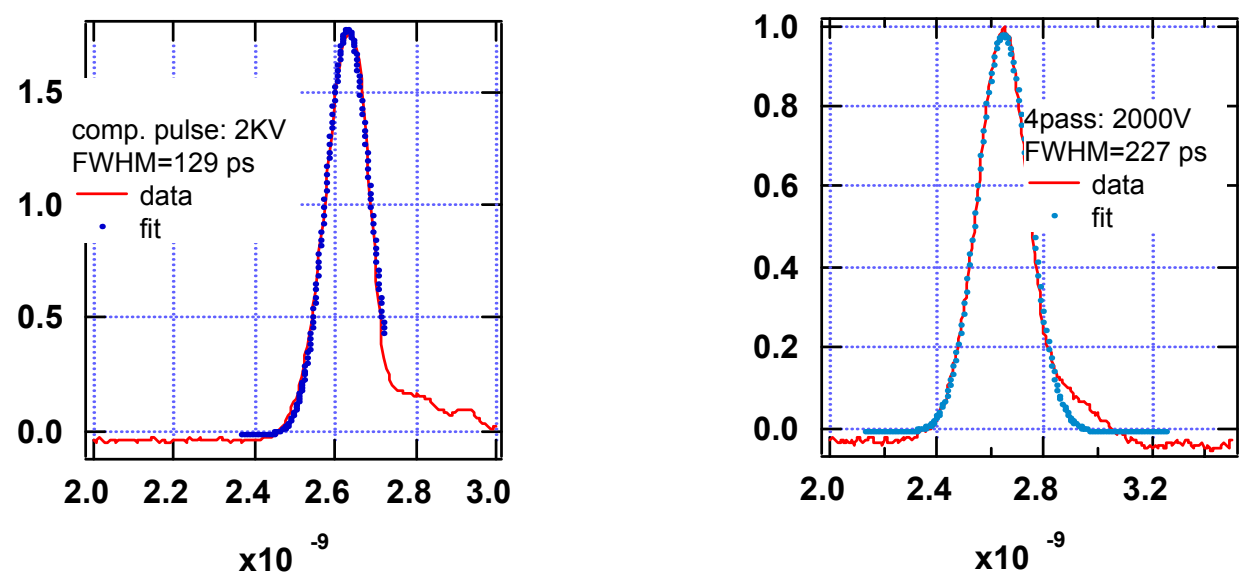

Figure 23. Pulse shapes measured at the compressor and 4-pass outputs using a $4.5 \mathrm{GHz}$ transient digitizer.

We recorded the compressed pulse shape at the output of the grating compressor as a means for obtaining the instrument function of the combined digitizer and photodiode, since the pulse at this location is much narrower than the combined BW of the detection 
system. The actual pulsewidth at the 4-pass output is the deconvolution of the measured pulsewidth and the instrument response width. For a Gaussian shaped pulse the actual pulse width is given by:

$$
\mathrm{T}_{\text {actual }}=\left\{\left(\mathrm{T}_{\text {meas }}\right)^{2}-\left(\mathrm{T}_{\text {instr }}\right)^{2}\right\}^{1 / 2} .
$$

and yields a value of $184 \mathrm{ps}$ for the 4-pass output pulsewidth.

\section{F. Autocorrelation measurement at the compressor output}

We measured the pulsewidth at the output of the grating compressor using a single-shot autocorrelator. In order to optimize the operation of the photogun we must be able to adjust the pulsewidth of the UV laser to produce a low emittance electron pulse while keeping the electron pulse length as short as possible. The desired pulse length of the UV pulse is a few ps, which can be obtained by varying the distance between the gratings in the compressor. We attempted to directly measure pulsewidth vs grating separation but found that the autocorrelator only gives good results for pulses $\sim 1$ ps or less, consequently we estimate the pulsewidth for $\mathrm{T}>1 \mathrm{ps}$ using various techniques. For pulse lengths $<1$ ps the autocorrelator appears to give accurate results. Figure 24 shows an autocorrelation for the case where the gratings separation has been set to give the shortest pulse.
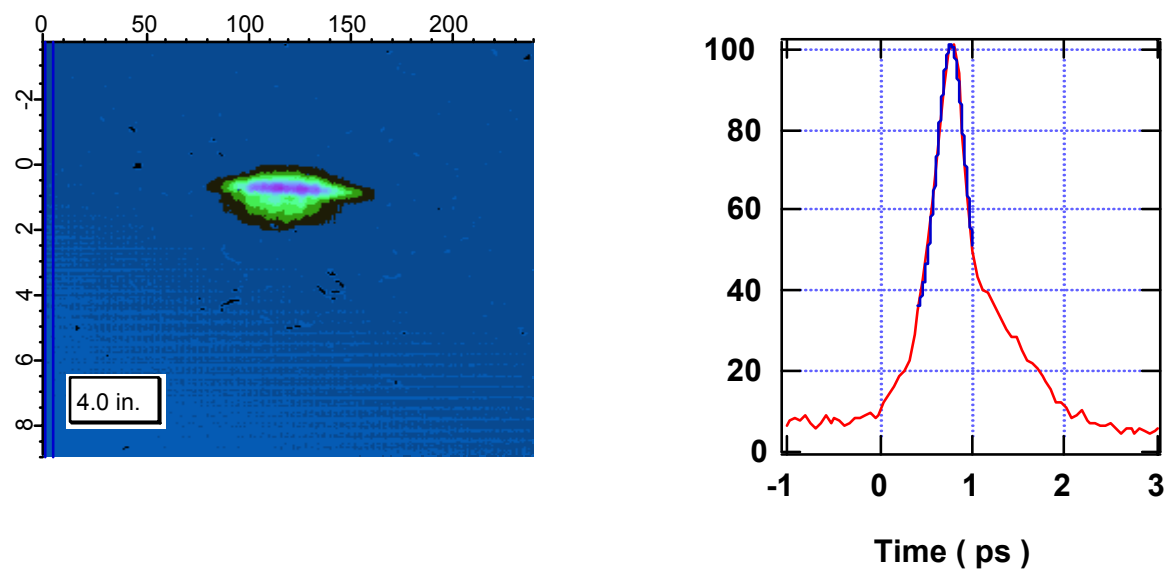

Figure 24. Image from single-shot autocorrelator and vertical lineout giving the pulsewidth, $\mathrm{Tp}=210 \mathrm{fs}$.

This data set shows a 210 fs pulse, the shortest pulse we measured was 150 fs which corrseponds to a time-bandwidth-limited pulse. We need to determine if the pedestal seen on the pulse in the image and lineout is real or an artifact of the measurement. To accurately measure pulses longer than $1 \mathrm{ps}$ we will build a special type of autocorrelator that uses a grating.

\section{PULSEWIDTH VERSUS GRATING SEPARATION}

\section{$\underline{\text { A. } 1 \omega \text { pulsewidth }}$}


By varying the distance between the gratings in the pulse compressor we can continuously adjust the pulse length from 184 ps to 150 fs. Figure 25 shows plots of the $1 \omega$ pulsewidth vs. grating separation. The three curves give the results of three different ways of modeling the compressor. One of the curves plots Eqn. 5a from Treacy's paper:[13]

$$
\Delta \mathrm{T}=\mathrm{b} \cdot(\mathrm{l} / \mathrm{d}) \cdot \Delta \lambda /(\mathrm{cd}) \cdot\left[1-((\mathrm{l} / \mathrm{d})-\sin \beta)^{2}\right]
$$

where $\mathrm{d}$ is the groove spacing of the gratings, $1 / 1480, \lambda$ is the center wavelength, and $\beta$ is the angle of the incident light with respect to the grating normal. The second curve is the input pulse width, $184 \mathrm{ps}$, minus the minimum pulse width, $150 \mathrm{fs}$, divided by the grating separation that produces the shortest pulse:

$$
\Delta \mathrm{T}=\{(184 \mathrm{ps}-0.15 \mathrm{ps}) / 864 \mathrm{~mm}\} \bullet \Delta \mathrm{b}
$$

where $\Delta b$, is the difference in grating separation from the minimum pulse length position.

The third curve in Figure 25 represents a Fourier transform-based model. A timebandwidth temporal pulse of $154 \mathrm{fs}$ is the input to a compressor of separation, $\mathrm{b}$. The temporal pulse is transformed to frequency space and multiplied by the term for the phase of the compressor, $\phi(\omega)$ :

$$
\begin{gathered}
\mathrm{E}(\omega, \Delta \mathrm{b})=\mathrm{E}(\omega, 0) \bullet \exp \{1 \phi(\omega)\} \\
\phi(\omega)=\beta_{0}+\beta_{1}\left(\omega-\omega_{0}\right)+\beta_{2}\left(\omega-\omega_{0}\right)^{2}+\beta_{3}\left(\omega-\omega_{0}\right)^{3}
\end{gathered}
$$

where $\phi(\omega)$ is given by Eqns. 11 and 17 in Treacy's paper.[13] $\phi(\omega)$ is a function of the grating deviation from the minimum pulsewidth spacing, $\Delta \mathrm{b}$, the grating groove density, $\mathrm{d}$, and the incident angle, $\alpha$. After multiplying by the phase, the field is inverse transformed back to the time-domain and converted to intensity to obtain a pulsewidth.

The models give similar results, $\Delta \mathrm{T} / \Delta \mathrm{b} \sim 0.2 \mathrm{ps} / \mathrm{mm}$, at $1 \omega$. As stated earlier the single-shot autocorrelator does not give accurate results beyond a $\sim 1$ ps pulsewidth. 


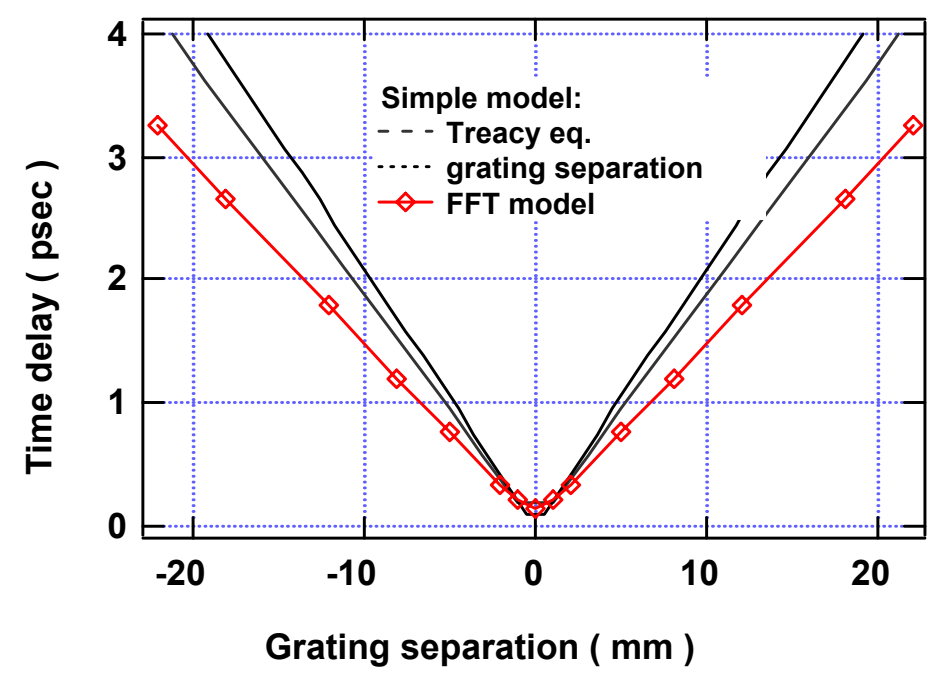

Figure 25. Plot of compressed pulsewidth versus grating separation. There are 2 data sets taken with the single-shot autocorrelator and 2 model curves.

\section{B. $3 \omega$ pulsewidth}

The $3 \omega$ intensity is proportional to the $1 \omega$ intensity, cubed. As a result the Gaussian-shaped pulse narrows up in time, i.e.,

$$
\mathrm{I}_{3 \omega}=\mathrm{I}_{03} \cdot \exp \left\{-4 \ln (2) \cdot\left[\left(\mathrm{t}-\mathrm{t}_{0}\right) / \mathrm{T}_{3 \omega}\right]^{2}\right\} \sim\left\{\mathrm{I}_{0} \bullet \exp \left\{-4 \ln (2) \cdot\left[\left(\mathrm{t}-\mathrm{t}_{0}\right) / \mathrm{T}_{3 \omega}\right]^{2}\right\}\right\}^{3}
$$

which yields the relationship,

$$
\mathrm{T}_{3 \omega}=\mathrm{T}_{1 \omega} / \operatorname{sqrt}(3) \text {. }
$$

Based on these results, the pulsewidth of the $3 \omega$ light is given:

$$
\begin{aligned}
\mathrm{T}_{3 \omega}=\{(0.2 \mathrm{ps} / \mathrm{mm}) / \mathrm{sqrt}(3)\} \bullet \Delta \mathrm{b} \\
=\{0.11 \mathrm{ps} / \mathrm{mm}\} \bullet \Delta \mathrm{b}
\end{aligned}
$$

where $\Delta b$, is the difference in grating separation from the minimum pulse length position. For example if the gratings are $100 \mathrm{~mm}$ from the minimum position, then the $3 \omega$ pulsewidth will be 11 ps.

\section{THOMSON X-RAY MEASUREMENTS}

The goal of the Falcon-Linac Thomson project is to develop a short-pulse, highbrightness, x-ray source for use as a probe of atomic and molecular dynamics in dense matter. At this stage we have built and tested the high-brightness photoinjector along with the UV photoinjector laser system. The Falcon laser system can deliver several $100 \mathrm{~mJ}$ 
pulses that upon compression are a few terawatts in power. Next year we will be moving the photoinjector onto the Linac and finish the beam transport line that brings Falcon laser pulses to the Linac vault for eventual interaction with the $100 \mathrm{Mev}$ electron pulse.

To produce $\sim 10^{9}$ Thomson x-rays we must accurately overlap the 35 fs laser pulse with the $\sim 5$ ps electron bunch in time and space. Part of the temporal synchronization between electrons and photons was solved by using UV light, seeded by the Falcon master oscillator, to produce photoelectrons at the cathode of the photoinjector. Next year we will phase-lock the master oscillator to the Linac rf to eliminate long term timing drift between laser and electrons. To spatially overlap the electrons and laser photons at the interaction point we must reduce laser pointing to $\sim 50 \mu \mathrm{rad}$. We will make careful measurements of pointing jitter and evaluate the need for an active control loop.

As an intermediate goal before moving the photoinjector onto the Linac, we attempted to generate Thomson $\mathrm{x}$-rays using the $5 \mathrm{MeV}$ electrons produced by the photoinjector. Since the beam transport system from Falcon is not complete, we will use $800 \mathrm{~nm}$ light from the PLS that is left over from frequency-tripling. Upon completion of the photoinjector characterization of the electron pulse energy, charge and emittance, we added a focusing solenoid magnet, electron-laser interaction chamber, bending magnet, and $\mathrm{x}$-ray detector for measuring Thomson $\mathrm{x}$-rays produced by scattering the unconverted $1 \omega$ light from the PLS with the focused $5 \mathrm{MeV}$ electrons. Figure 26 shows a layout of the photoinjector with interaction chamber, beam-dump magnet, focusing magnet, and $1 \mathrm{w}$ laser input.

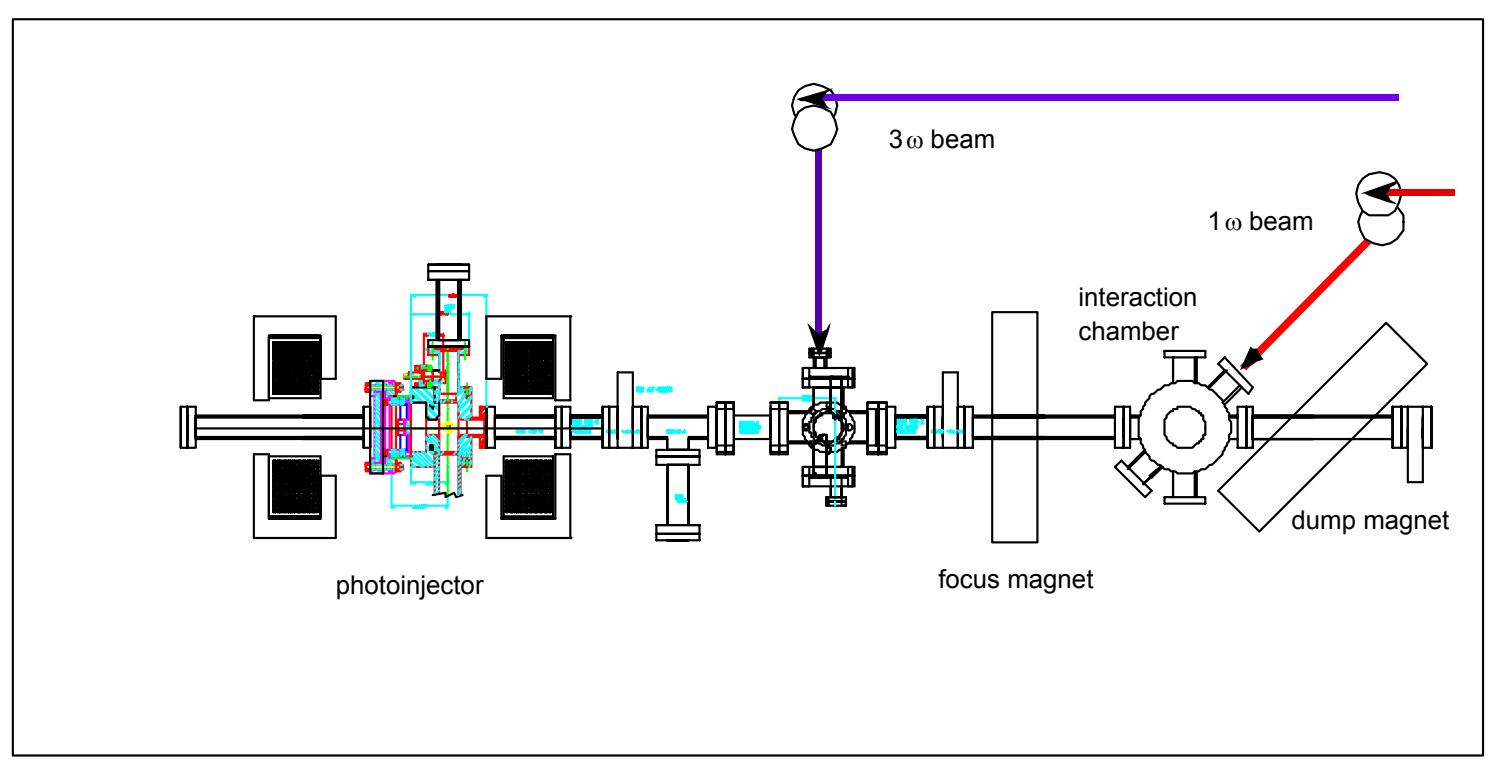

Figure 26. Layout for low energy Thomson x-ray measurement.

The $3 \omega$ and $1 \omega$ beams are separated at the output of the frequency conversion section of the PLS using dichroic mirrors. Both beams travel to the photoinjector table along roughly parallel beam paths as shown in Figure 27 . The $3 \mathrm{w}$ beam is raised to the level of the photocathode and electron beam path using a periscope as shown in Fig. 26. The UV beam passes through a fused silica port and is deflected to the copper photocathode with a 45 degree turning mirror that is mounted inside the vacuum 
chamber. The parallel $1 \mathrm{w}$ beam path is sent directly to the interaction chamber as shown in the figure. The $1 \mathrm{w}$ and $3 \mathrm{w}$ beams derive from the same pulse at the PLS, but travel along different paths once they reach the photoinjector table: the $3 \mathrm{w}$ pulse travels an additional distance to reach the cathode and the accelerated electrons travel from the cathode to the interaction region. To accommodate the additional distance traveled by the UV pulse and the electrons we added an adjustable delay leg to the $1 \mathrm{w}$ beam path on the PLS table (see Fig. 27). We can adjust the pathlength of the $1 \mathrm{w}$ light so that the electrons and laser photons arrive at the path intersection in the interaction chamber at the same time.

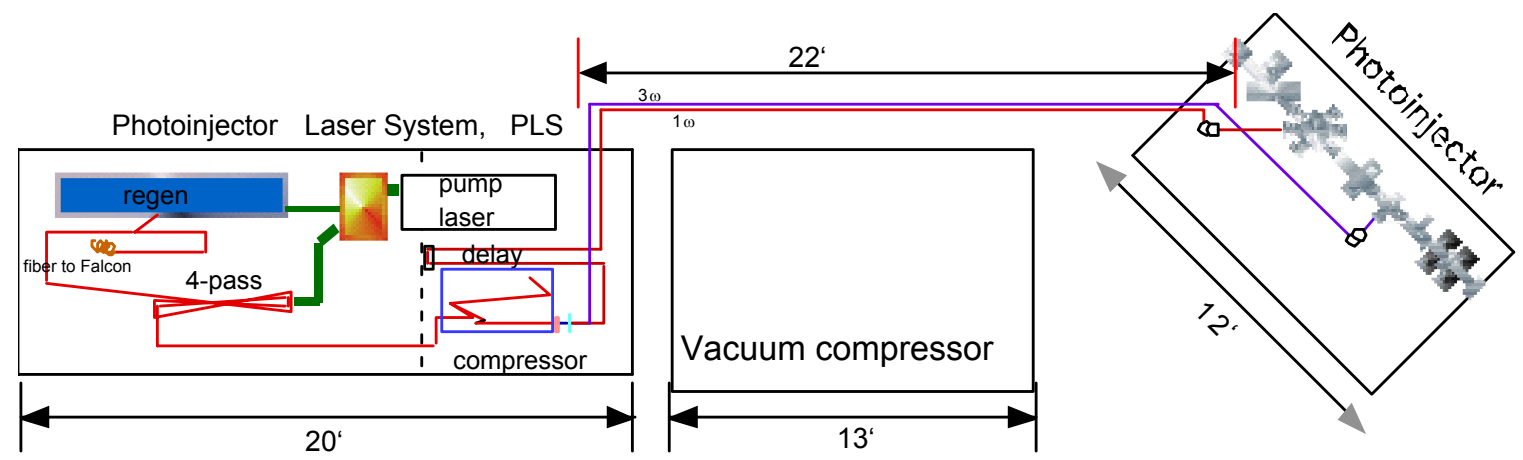

Figure 27. Layout of photoinjector lab in B194 basement shows relative size and location of major systems and beam path for $1 \mathrm{w}$ and $3 \mathrm{w}$ light.

The electron beam emerging from the photogun is focused by a large solenoid magnet to a waist at the center of the interaction chamber. The $1 \mathrm{w}$ laser pulse is focused with a $300 \mathrm{~mm}$ lens and enters the interaction chamber through a port at an angle of 135 degrees. A pop-in scintillator is mounted on a port at 90 degrees with respect to the ebeam axis and allows a thin aluminized, Ce-doped YAG crystal to be placed at chamber center where the laser beam and e-beam are intended to intersect. We use the top port of the interaction chamber to mount cameras and photodetectors for diagnosing the spatial and temporal overlap between electron and laser pulses. Figure 28 shows a layout of the interaction chamber.

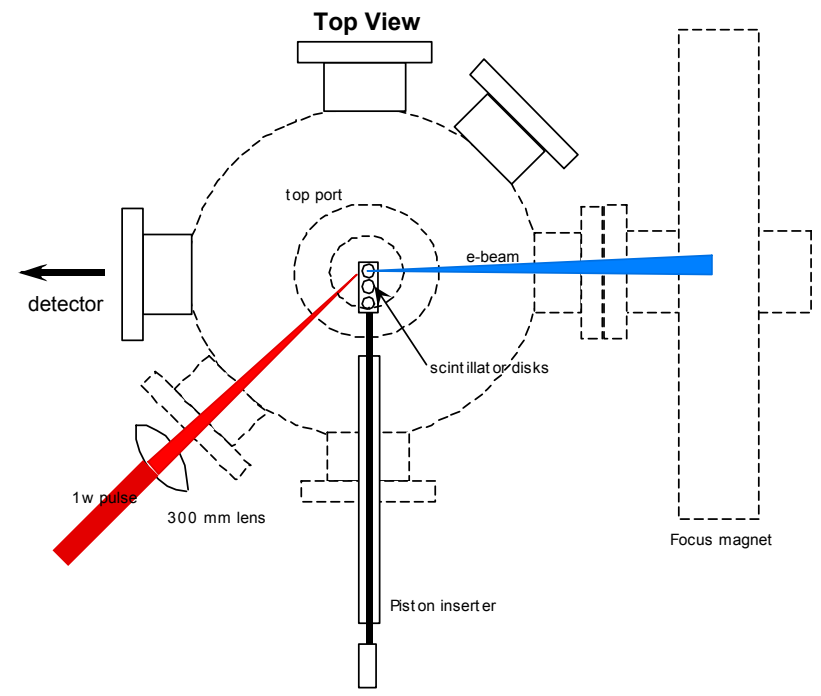


Figure 28. Top view of the target interaction chamber shows the inserter for placing a scintillator at the chamber center and viewing the spatial overlap between the laser pulse and electron beam.

To overlap the laser and e-beam spatially we reduce the laser intensity and insert a scintillator disk at the chamber center. We use two cameras mounted to view the scintillator through the top port. One camera views a large field that includes the entire scintillator disk while a second camera magnifies the overlap region so that the focused spot fills the entire camera field. We use a fast photomultiplier to look at the temporal signals from the scintillator due to the laser pulse or e-beam induced fluorescence. We cannot precisely overlap two picosecond pulses using a photomultiplier and oscilloscope so we find a region of overlap between the two signals. First we set the delay leg on the PLS table (see Fig. 27) so that one pulse, e.g. the laser light, leads the scintillator signal by a discernible amount. We mark the corresponding position on the delay rail. Next we change the delay until the other signal is in front and mark this position on the rail. These two positions bracket the region of overlap between the electron and laser pulses, and we look for Thomson x-rays in this region.

After the interaction chamber the electrons are deflected into a dump tank using a permanent magnet as shown in Fig. 26. The x-rays travel to the end of the beamline where we have mounted a MCP/CCD detector. In front of the detector we have a thin, aluminized mylar film that is opaque to visible light and about $30 \%$ transmissive to 500 $\mathrm{eV}$ x-rays. The input of the detector contains a single microchannel plate (MCP) that is coated with CsI to convert the x-rays to photoelectrons. The MCP is biased at $1 \mathrm{kV}$ and has an electron multiplication factor of $\sim 10^{4}$. The electrons emerging from the MCP are accelerated to $3.5 \mathrm{kV}$ and slam into a phosphor that converts the electron energy to visible light that is imaged with a CCD camera.

We can estimate the photon energy and number of Thomson x-rays based on our measured values of laser energy, e-beam charge, electron energy, spatial overlap, and temporal pulse length. The energy of the Thomson x-rays is given by:[14]

$$
\boldsymbol{E}=\boldsymbol{E}_{\text {laser }} \cdot 2 \gamma^{2} \frac{(1-\cos \Psi)}{\left(1+\gamma^{2} \theta_{x}^{2}\right)}
$$

where $\mathrm{E}$ is the energy of the $\mathrm{x}$-ray photons, $\mathrm{E}_{\text {laser, }}$, the energy of the $1 \mathrm{w}$ laser photons, $\gamma$ is the ratio of the electron kinetic energy to the rest mass, $\gamma=9.8$ for $5 \mathrm{Mev}$ photons, $\Psi$ is the angle between the e-beam and laser propagation directions, and $\theta_{\mathrm{x}}$ is the observation angle with respect to the electron propagation axis. Figure 29 shows a cartoon of the interaction region, showing the angles used in Eq. (9). 


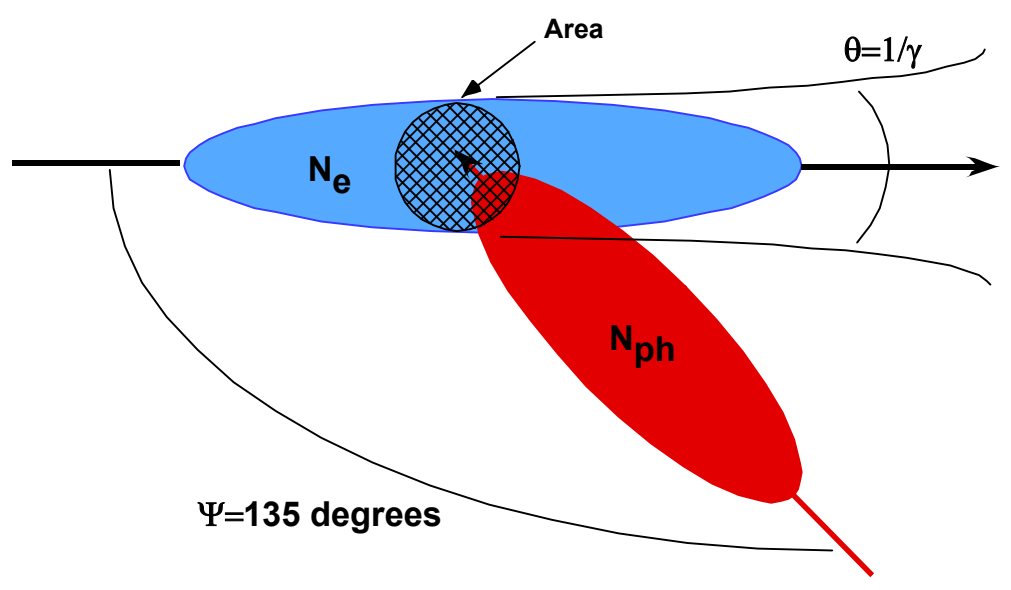

Figure 29. The cartoon shows the interaction between the electron beam pulse, $\mathrm{N}_{\mathrm{e}}$, and the $1 \omega$ laser pulse, $\mathrm{N}_{\mathrm{ph}}$, at a collision angle, $\Psi=135$ degrees.

The number of Thomson photons generated by the interaction depicted in Fig. 29 is the product of the number of electrons, $\mathrm{N}_{\mathrm{e}}$, and $1 \mathrm{w}$ laser photons, $\mathrm{N}_{\mathrm{ph}}$, that interact in the Area of intersection:[14]

$$
N_{x-\text { rays }} \approx \frac{N_{1 w} \cdot N_{e}}{\operatorname{Area}} \frac{\tau_{\text {laser }}}{\tau_{e}} \sigma_{T}
$$

The other quantities in Eq. 10 are the temporal lengths of the laser and electron pulses, and the Thomson scattering cross-section, $\sigma_{\mathrm{T}}=(8 \pi / 3) \mathrm{r}_{\mathrm{e}}{ }^{2}=6.6 \times 10^{-25} \mathrm{~cm}^{2}$, where $\mathrm{r}_{\mathrm{e}}$ is the classical electron radius. Table 7 gives the estimated or measured values for the quantities in Eqs. 9 and10, along with the resulting number of Thomson x-rays. The estimate for the Thomson x-rays is an upper bound because we have assumed perfect spatial and temporal overlap between the electron and photon pulses.

1. Laser pulse energy

2. $1 \mathrm{w}$ photon energy

3. Number of $1 \mathrm{w}$ photons

4. Electron beam charge

5. Electron energy

6. Relativistic gamma

7. Number of electrons

8 . Focal spot of laser (1/ $\mathrm{e}^{2}$ radius)

9. Width of electron bunch @ focus

10. Intersection area

11. $1 \mathrm{w}$ laser pulse length

12. Electron bunch length

13. Intersection angle

14. Number of Thomson x-rays

15. X-ray divergence angle, $1 / \gamma$,

16. X-ray photon energy
$5 \mathrm{~mJ}$

$1.55 \mathrm{eV}$

$2.01 \times 10^{16}$

$0.4 \mathrm{nC}$

$5 \mathrm{MeV}$

9.8

$2.5 \times 10^{9}$

$50 \mu \mathrm{m}$

$50 \mu \mathrm{m}$

$3.9 \times 10^{-5} \mathrm{~cm}^{2}$

$7.1 \mathrm{ps}$

$5.5 \mathrm{ps}$

135 degrees

$6.6 \times 10^{5}$

$102 \mathrm{mrad}$

$<520 \mathrm{eV}$ 
Table 7. List of the parameters relevant to estimating the number of Thomson x-rays produced in the interaction region.

According to the numbers in Table 7 , we could produce $6.6 \times 10^{5}$ Thomson photons in the interaction region if we achieve perfect spatial and temporal overlap between the photon and electron pulses and we match the laser and electron focal spot areas. A more relevant quantity for the experiment is the number of Thomson x-rays that appear at the detector, and what is the signal-to-noise ratio at the detector. To calculate the number and energy distribution of Thomson x-rays at the plane of the MCP we use Eqs. 9 and 10 to generate a $2 \mathrm{D}$ contour map showing x-ray energy and number as a function of radial position at the MCP detector. Figure 30 shows a sketch of the x-ray divergence from the interaction region to the plane of the MCP, located $47 \mathrm{~cm}$ downstream. The MCP is 25 $\mathrm{mm}$ in diameter and subtends a $53 \mathrm{mrad}$ angle, whereas the Thomson x-rays diverge at $1 / \gamma=102 \mathrm{mrad}$. The image in the lower half of Figure 30 shows the distribution of x-ray photons in number and energy (contours), based on Eqs. 9 and 10. The inner circle on the image plot represents the MCP detector, assuming perfect pointing of the x-rays to the detector.
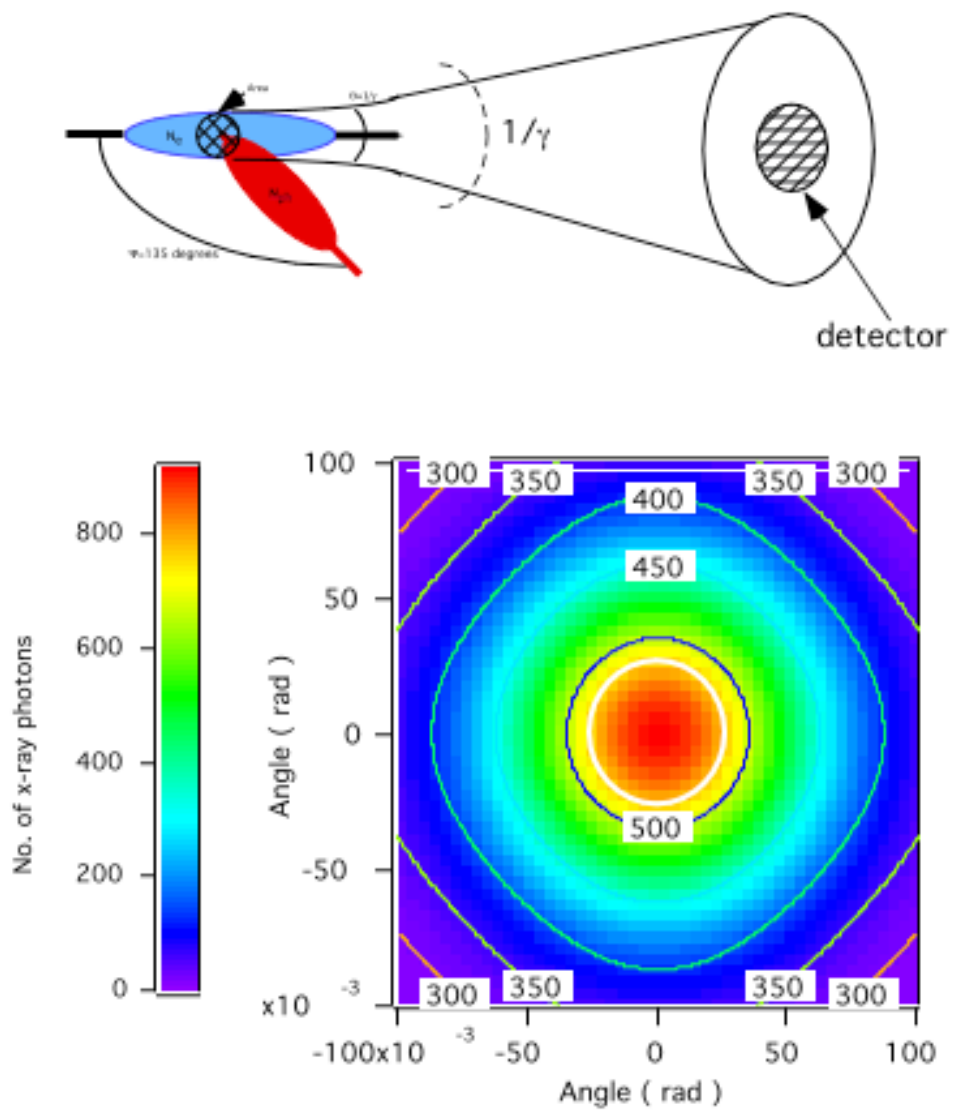

Figure 30. The top sketch shows the interaction region, the divergence of the x-rays and the area subtended by the MCP/CCP detector. The lower image shows the particle number distribution at the detector plane and contours giving the photon energy distribution at the detector plane in $\mathrm{eV}$. 
To determine the actual signal produced from the number of x-rays at the plane of the $\mathrm{MCP}$ detector we must account for the efficiency for converting the x-rays to detector signal. We placed a thin aluminized film in front of our detector to block visible photons and pass the $500 \mathrm{eV}$ x-rays. The film is composed of $0.3 \mu \mathrm{m} \mathrm{Al}$ deposited on $0.5 \mu \mathrm{m}$ of mylar. The transmission of this composite film as a function of x-ray energy [15] is shown in Fig. 31. The MCP detector is coated with $\sim 0.3 \mathrm{~mm}$ of CsI, a material that converts the $\mathrm{x}$-rays to electrons for amplification by the MCP. Based on the manufacturers data, the quantum efficiency of the CsI is $\sim 0.4$ for converting $500 \mathrm{eV}$ photons into photoelectrons.[16] Table 8 summarizes the various factors that contribute to converting $\mathrm{x}$-rays to signal in our current measurement scheme. Under the ideal conditions of perfect spatial and temporal overlap of the electron and laser pulses, and perfect pointing alignment of the Thomson x-rays with the MCP/CCD detector, we should see an integrated signal of $5.4 \times 10^{9}$ counts. In the next section we will describe our experimental results.

1. Total number of $\mathrm{x}$-rays produced

2. Fraction of $x$-rays subtended by the detector

3. Transmission of Al film

4. Quantum efficiency of CsI coating

5. Net X-ray to photoelectron conversion

6. Fraction of MCP area that produces electrons

7. Number of photoelectrons at MCP input

8. Number of pixels in CCD array

9. Fraction of pixels illuminated

10. Number of shots required for full illumination

11. Time required for full detector illumination @ $10 \mathrm{~Hz}$

12. MCP gain

13. phosphor conversion efficiency

13. Integrated $\mathrm{x}$-ray counts after $60 \mathrm{sec}$
$6.6 \times 10^{5}$
0.14
0.3
0.4
0.017
0.4
$4.5 \times 10^{3}$
$3.43 \times 10^{5}$
$1.3 \times 10^{-2}$
76
$7.6 \mathrm{sec}$
$10^{4}$
0.2
$5.4 \times 10^{9}$

Table 8. Signal analysis for MCP/CCD detector

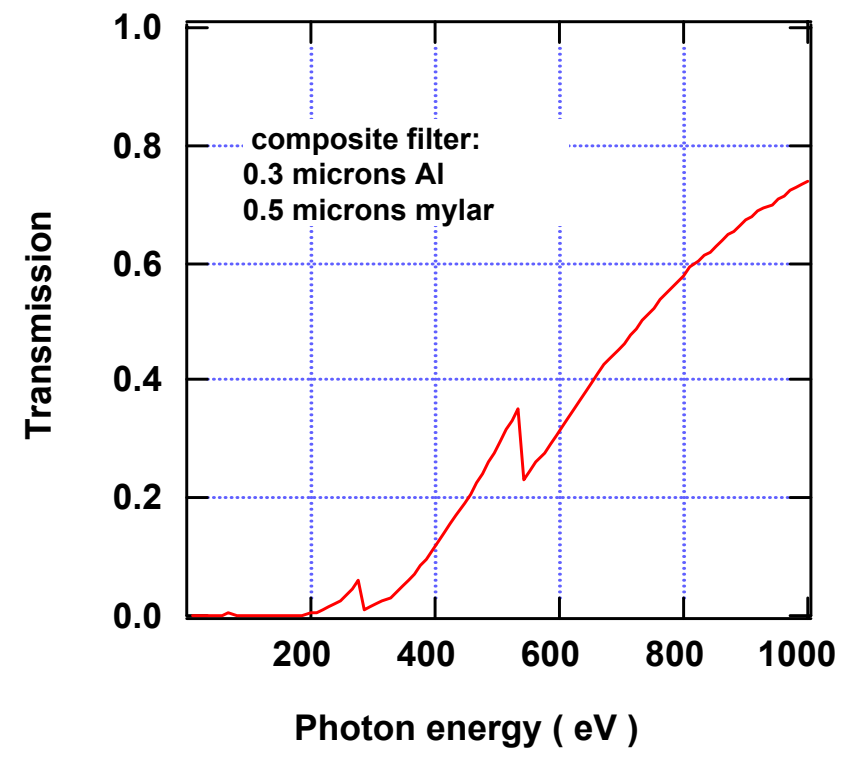


Figure 31. This plot shows the transmission as a function of x-ray energy of the aluminized mylar film.

\section{RESULTS}

We took a series of integrated 60 second shots with our MCP/CCD detector to try and detect Thomson x-rays. For each shot we slowly translated the delay stage of our rail $\sim 25 \mathrm{~mm}$ to try and move the $1 \mathrm{w}$ pulse into overlap with the electron pulse. The MCP was gated with a $700 \mathrm{~V}, 100 \mathrm{~ns}$ pulse, to provide gain to the MCP only when photoelectrons are produced. A $700 \mathrm{~V}$ pulse produces an electron multiplication factor of $\sim 200$ compared with a value of $10^{4}$ for a bias voltage of $1 \mathrm{KV}$. Figure 32 shows a typical image from the $\mathrm{MCP} / \mathrm{CCD}$ detector and a plot of the integrated counts per 60 second exposure as a function of translation stage position. As seen by this plot the signal generated from background x-rays produced by the photoelectron bunch is $\sim 20 \mathrm{X} 10^{9}$ counts. If we account for the lower gain from the $700 \mathrm{~V}$ bias, the maximum signal-tonoise we can expect to achieve from this measurement technique, based on our signal estimate from Table 8 , is $\left(5.4 \times 10^{9} / 20 \times 10^{9}\right) *\left(200 / 10^{4}\right)=5.4 \times 10^{-3}$. This value for signalto-noise estimate is based upon perfect spatial and temporal overlap between electrons and photons in the interaction region and perfect pointing of the X-rays at the detector. In reality our $\mathrm{S} / \mathrm{N}$ estimate could be much lower than $5.4 \times 10^{-3}$.
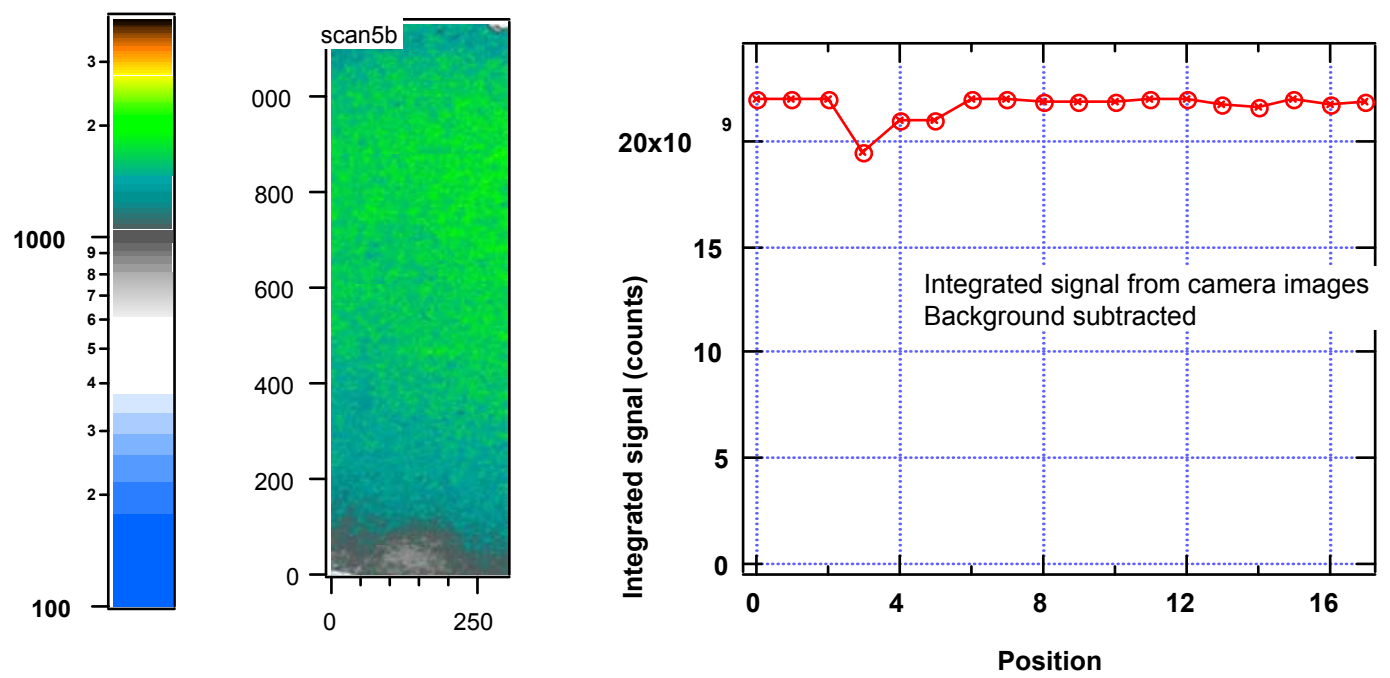

Figure 32. Image from MCP/CCD camera is shown with color table. The plot shows the integrated signal for several such camera images plotted against the translation stage position in inches.

To increase the signal-to-noise ratio of our measurement of $500 \mathrm{eV}$ Thomson $\mathrm{x}-$ rays we need to either reduce or discriminate against the large background produced by the $5 \mathrm{MeV}$ electrons. These electrons produce hard x-rays whenever they collide with the walls of the vacuum chamber or other solid objects in the system. Although we believe that we dump most of the electron bunch in the dump tank, these dumped electrons may also be a large source of background x-rays. One technique for distinguishing the $500 \mathrm{eV}$ 
Thomson x-rays from the harder, background x-rays, is to employ single-photon counting techniques. We will use a liquid-nitrogen-cooled, $\mathrm{Si}-\mathrm{Li}$, $\mathrm{x}$-ray detector that is sensitive to single photons. We plan to place this detector several feet behind the location of the current $\mathrm{MCP} / \mathrm{CCD}$ detector and shield it with lead bricks to reduce the background $\mathrm{x}$-ray noise. If we can reduce the background sufficiently to use single-photon detection we can produce a histogram of number of counts vs. counts/event that can be calibrated to yield an energy spectrum of detected x-rays. This technique should alllow us to discriminate the Thomson x-rays from the hard x-rays based upon energy. We are currently working on this measurement.

\section{SUMMARY}

This report discusses progress made in the past year toward developing a ultrafast, hard x-ray source based upon Thomson scattering of laser photons from relativistic electrons. Our ultimate goal is to scatter a 4 Joule, 35 fs pulse from a $100 \mathrm{MeV}, 1 \mathrm{nC}$ electron bunch to produce $\sim 10^{9}$ photons at $\sim 100 \mathrm{keV}$ energy. In the last year we have made important progress toward this goal by completing construction and testing of the 5 Mev photoinjector gun and the UV photoinjector laser system. We attempted an experiment to produce and diagnose soft Thomson x-rays generated by scattering a weak $800 \mathrm{~nm}$ laser pulse from the $5 \mathrm{Mev}$ photoinjector electron bunch. This experiment has given us valuable experience in constructing the interaction chamber, diagnosing spatial and temporal overlap between the electron and laser pulses, and detecting Thomson $\mathrm{x}$ rays against a large background of hard $\mathrm{x}$-rays produced from the scattered $5 \mathrm{Mev}$ electrons. In the coming year we will place the photoinjector on the Linac and measure the characteristics of the $100 \mathrm{Mev}$ electron bunch. In a parallel effort we will upgrade the Falcon laser system: first to $10 \mathrm{TW}$ and then to $100 \mathrm{TW}$. In the following year we will construct the interaction chamber at the Linac output and bring the $100 \mathrm{TW}$ laser pulse to collide with the $100 \mathrm{MeV}$ electron bunch to produce an ultrafast, hard x-rays light source for dynamic, laser-matter interaction experiments at solid densities.

This work was performed under the auspices of the U.S. Department of Energy by University of California, Lawrence Livermore National Laboratory under contract No. W-7405-Eng-48.

\section{REFERENCES}

1. "Low Emittance, High Brightness: A New X-Ray Light Source", Science \& Technology Review, UCRL-52000-00-9, pp.23-25, Sept. 2000.

2. Todd Ditmire, "Ultrafast Materials Probing with the Falcon/Linac Thomson X-ray Source", Strategic Initiative Proposal, March, 2000.

3. G. R. Hays, T. Cowan, J. K. Crane, G. P. LeSage, M. D. Perry, H. T. Powell, M. Roth, K. B. Wharton, V. P. Yanovsky, "Progress toward an integrated $100 \mathrm{TW}$ laser-100 MeV electron linac experiment", Technical Digest CLEO 2000 (Conf. on Lasers and Electro-Optics) paper CWK9

4. G. P. LeSage, S. G. Anderson, T. E. Cowan, J. K. Crane, T. Ditmire and J. B. Rosenzweig, "RF Photoinjector Development for a Short-Pulse, Hard X-ray Thomson Scattering Source", 
Proceedings on the Advanced Accelerator Conference 2000, (to be published through AIP Press).

5. D.T. Palmer et al., "Emittance studies of the BNL/SLAC/UCLA 1.6 cell photocathode rf gun," IEEE Part. Accel. Conf. (1997).

6. H. Matsumoto et al., "Applications of hot isostatic pressing (HIP) for high gradient accelerator structure,” Proc. PAC '91 1008 (1992).

7. POISSON/SUPERFISH computer codes developed at LANL, LA-UR-96-1834.

8. D. Gooden and J. Rosenzweig, "Modeling of the x-ray radiation dependence on power in high gradient radio-frequency accelerator structures," UCLA Part. Beam Phys. Lab. Internal Report (1999).

9. X.-J. Wang et al., "Intense electron emission due to picosecond laser produced plasmas in high gradient electric fields," Journal of Applied Physics, 72(3), p. 888-894 (1992).

10. J. Rosenzweig and G. Travish, "Design Considerations for the UCLA PBPL Slit-based Phase Space Measurement Systems," Nucl. Instrum. Meth. A 341, p. 379 (1994).

11. A. E. Siegman, Lasers, pp. 746-749, University Science Books 1986.

12. A. Brun, P. Georges, G. Le Saux, and F. Salin, "Single-shot characterization of ultrashort light pulses",

13. E. B. Treacy "Optical Pulse Compression With Diffraction Gratings", J. Quant. Electronics, QE-5, 454-458, (1969).

14. R. W. Schoenlein, W. P. Leemans, A. H. Chin, P. Volfbeyen, T. E. Glover, P. Balling, M. Zolotorev, K. -J. Kim, S. Chattopadhyay, "Femtosecond X-ray Pulses at 0.4 A Generated by $90 \mathrm{deg}$ Thomson Scattering: A Tool for Probing the Structural Dynamics of Materials", Science, 274, 236, (1997).

15. We obtained transmission values for the aluminized films from the website for the $x$-ray optics group at Lawrence Berkeley Laboratory: http://www.cxro.lbl.gov.

16. The quantum efficiency for CsI coated MCPs was found in "Long-Life Microchannel Plates", a publication from Burle Technologies, Inc. 